JOURNAL INTERNATIONAL DE TECHNOLOGIE, DE L'INNOVATION, DE LA PHYSIQUE, DE L'ENERGIE ET DE L'ENVIRONNEMENT

\title{
Calcul de la permittivité dans un plasma d'air hors de l'équilibre chimique et thermique : effet sur la propagation des ondes électromagnétiques à différentes altitudes
}

P. André, S. Lalléchère

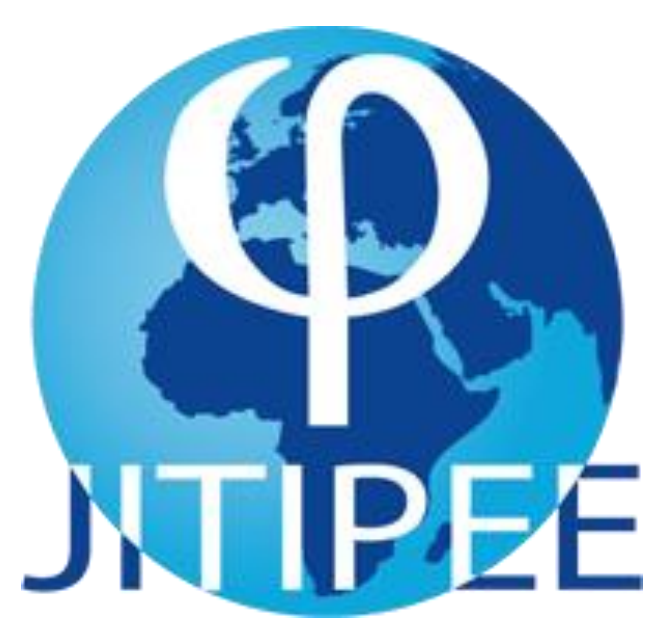

ISSN : 2428-8500

DOI : 10.52497/jitipee.v7i1.286 


\title{
Calcul de la permittivité dans un plasma d'air hors de l'équilibre chimique et thermique : effet sur la propagation des ondes électromagnétiques à différentes altitudes
}

\author{
Pascal André ${ }^{(1)}$, Sébastien Lalléchère ${ }^{(2)}$ \\ (1) Université Clermont Auvergne, CNRS, Laboratoire de Physique de Clermont, F-63000 \\ CLERMONT-FERRAND, FRANCE \\ (2) Université Clermont Auvergne, CNRS, SIGMA Clermont, Institut Pascal, F-63000 \\ CLERMONT-FERRAND, FRANCE \\ pascal.andre@uca.fr
}

\begin{abstract}
Résumé - Dans cet article, nous rappelons l'ensemble des formulations nécessaire aux calculs des concentrations des espèces chimiques et de la permittivité dans un plasma d'air à l'équilibre et hors de l'équilibre thermique en fonction du temps. Nous discutons du calcul des fréquences de collision des électrons et des espèces neutres $\left(\mathrm{O}, \mathrm{N}, \mathrm{O}_{2}, \mathrm{~N}_{2}, \mathrm{NO}\right)$ individuelles et leur influence sur le calcul de la permittivité du milieu. Puis, nous présentons les résultats de la permittivité diélectrique du milieu correspondant à deux altitudes $(0 \mathrm{~km}$ et $10 \mathrm{~km})$ et pour deux déséquilibres thermiques $(\theta=1$ et $\theta=2)$. Le plasma ainsi étudié pouvant être rapproché de ceux produits lors de la foudre, du brouillage électromagnétique, des plasmas de rentrée atmosphérique, des phénomènes liés à la furtivité... L'une des originalités de ces travaux repose également sur la présentation des résultats, à la fois en fonction de la fréquence de l'onde incidente et du temps, ceci permettant d'accéder à la dynamique comportementale du milieu plasma. Finalement, nous appliquons les résultats obtenus à deux configurations de test. Le premier considère une couche de plasma insérée entre deux espaces libres et le second considère une couche de plasma accolée à une plaque de métal parfait. Finalement, nous présentons les calculs de la transmission et de la réflexion d'une onde incidente dans ces deux cas et pour le plasma considéré. Du point de vue de ces propriétés électromagnétiques, nous montrons que le plasma peut jouer le rôle d'un métamatériau.
\end{abstract}

Mots clés : équilibre chimique, équilibre thermique, onde électromagnétique, plasma, réflexion, transmission, foudre, furtivité, brouillage électromagnétique.

DOI : 10.52497/jitipee.v7i1.286 


\section{Introduction}

Dans de nombreux systèmes développés ou en développement, une meilleure compréhension de l'interaction d'une onde électromagnétique et d'un plasma peut permettre de lever certains verrous technologiques. Nous pouvons citer l'interaction des systèmes embarqués avec le plasma de l'onde de choc lors de rentrée atmosphérique [1], les phénomènes de furtivité d'engins hypersoniques [2], le brouillage d'onde électromagnétique lors d'impulsion électromagnétique [3], les perturbations électromagnétiques lors du déclenchement de la foudre et le besoin de systèmes de protection [4], des problèmes liés à la création d'un plasma dans le guide d'onde pouvant servir à la protection du duplexeur d'une antenne [5]...

Lors de la rentrée atmosphérique d'un objet artificiel, le plasma se trouvant derrière l'onde de choc est fortement déséquilibré thermiquement et chimiquement [6]. Dans le cas de l'arc de foudre, l'explosion provoquée durant la première phase de la création du plasma est suffisamment rapide pour supposer qu'aucun échange d'énergie n'ait lieu avec l'extérieur [7]. De plus, dans notre étude, nous supposons que le gaz atteint immédiatement une température donnée ou des températures données suivant la rapidité de la thermalisation qui dépend des types de choc entre particules [8]. Puis, les processus de réaction sont mis en place pour atteindre l'état d'équilibre chimique. Par conséquent, nous pouvons noter que le plasma peut se trouver dans différents états thermodynamiques allant de l'équilibre thermique et chimique au hors de l'équilibre.

L'étude de l'interaction des ondes électromagnétiques dans tout milieu matériel (plasma dans cette étude) nécessite la définition de conditions et hypothèses spécifiques (e.g., diffusion ou non des particules, phénomènes de convection, présence ou non de dipôles créés par l'onde électromagnétique incidente, plasma (non-)magnétique, etc.). Ces hypothèses seront évaluées et mises en relation avec les applications potentielles visées : foudre, rentrée atmosphérique, furtivité, brouillage électromagnétique, ...

Pour étudier l'interaction d'une onde électromagnétique et de son environnement, la permittivité du milieu est l'un des paramètres clefs [9]. Récemment de nombreuses publications tendent à montrer que les plasmas dans certains cas peuvent devenir des métamatériaux de type main gauche [10].

Dans cet article, afin d'étudier, la permittivité du milieu plasma et de son évolution au cours du temps, nous prenons en considération quatre plasmas différents hors de l'équilibre chimique. Ainsi, nous étudions deux plasmas à la pression au niveau de la mer soit une pression de $101325 \mathrm{~Pa}$., le premier à l'équilibre thermique et le second hors de l'équilibre et deux plasmas à la pression qui correspond à une altitude de $10 \mathrm{~km}$ soit une pression de $26442 \mathrm{~Pa}$., le premier à l'équilibre thermique et le second hors de l'équilibre. La composition initiale que nous considérons est de $79 \%$ de diazote $\mathrm{N}_{2}$ et $21 \%$ dioxygène $\mathrm{O}_{2}$. Dans un premier paragraphe, nous étudions les formulations de base permettant de calculer la cinétique chimique puis les formulations permettant de calculer les permittivités en fonction de la fréquence de l'onde incidente. Puis, dans un second paragraphe, nous définissons deux cas de test. Le premier cas de test, test \#1, considère une couche de plasma insérée entre deux espaces libres et le second cas de test, test \#2, considère une couche de plasma accolée à une plaque de métal parfait. Ces deux cas de test nous permettent de mettre en exergue l'influence de la permittivité sur la transmission et la réflexion d'une onde incidente électromagnétique. 


\section{Formulations et bases de données}

\subsection{Calcul des concentrations chimiques}

Le plasma étant en évolution dans le temps, il est le siège de réactions chimiques permettant son passage de l'état initial à l'état final. Pour une réaction chimique $l$ donnée, la vitesse d'avancement de la réaction s'écrit :

$$
\frac{d \xi_{l}}{d t}=k_{d}^{l} \prod_{j}^{N} n_{j}^{v_{d j}^{l}}-k_{i}^{l} \prod_{j}^{N} n_{j}^{v_{i j}^{l}}
$$

Avec $k_{d}^{l}$ et $k_{i}^{l}$ les taux de réaction de la réaction chimique $l$ respectivement dans le sens direct et inverse, $v_{d j}^{l}$ et $v_{i j}^{l}$ les coefficients stœchiométriques de l'espèce chimique $j$ respectivement pour les réactifs (elle est égale à zéro si l'espèce n'est pas présente) et pour les produits, $n_{j}$ désigne la concentration de l'espèce chimique $j, \xi_{l}$ est le coefficient d'avancement de la réaction $l$ et $N$ est le nombre d'espèces chimiques prises en compte.

Lorsque l'équilibre chimique est atteint, si les températures sont stationnaires et en l'absence de diffusion et de convection, les vitesses d'avancement pour toutes les réactions chimiques $l$ sont nulles. On obtient alors :

$$
\frac{d \xi_{l}}{d t}=0 \text { pour } l \in\left[1 . . N_{r}\right]
$$

Avec $N_{r}$ le nombre de réactions prises en compte.

Ou, autrement dit, la vitesse de la réaction directe $k_{d}^{l} \prod_{j}^{N} n_{j}^{v_{d j}^{l}}$ est égale à la vitesse de la réaction inverse $k_{i}^{l} \prod_{j}^{N} n_{j}^{v_{i j}^{l}}$. Ainsi avec la relation (1), on obtient la relation suivante :

$$
k_{i}^{l}=k_{d}^{l} \prod_{j}^{N} n_{j}^{v_{d j}^{l}-v_{i j}^{l}}=\frac{k_{d}^{l}}{K}
$$

Cette relation permet de calculer le taux de réaction inverse en fonction du taux de réaction directe si on connait soient les concentrations chimique $n_{j}$ soit la constante d'équilibre $K$. Nous ne pouvons pas prendre les taux de réaction inverse et direct directement dans la littérature. En effet, les taux de réaction sont souvent mal connus et doivent être choisis avec précaution [11] et il est nécessaire d'obtenir la concentration de l'équilibre chimique lorsque le mélange est stable dans le temps. C'est-à-dire au bout d'un temps suffisamment long. Par conséquent, afin de déterminer un jeu complet de taux de réaction, nous devons connaître la concentration chimique obtenue à l'équilibre chimique puis calculer le taux inverse ce qui garantit d'obtenir les concentrations souhaitées lorsque l'état stationnaire est atteint.

À l'équilibre chimique, nous supposons que les contraintes extérieures sont la pression et les températures, nous obtenons la concentration chimique en utilisant la minimisation de l'énergie libre de Gibbs (enthalpie libre) [12]. 
L'énergie libre de Gibbs, s'écrit :

$$
G=\sum_{j=1}^{N} n_{j}\left[\mu_{j}^{0}+k T_{j} \ln \left(\frac{T_{j} n_{j}}{\sum_{j=1}^{N} n_{j} T_{j}}\right)+k T_{j} \ln \left(\frac{P}{P^{0}}\right)\right]
$$

Où $T_{j}$ représente la température de translation de l'espèce chimique $j, P$ la pression, $P^{0}$ la pression standard $10^{5} \mathrm{~Pa}, k$ la constante de Boltzmann et $\mu_{j}^{0}$ est le potentiel chimique de l'espèce chimique $j$. Les potentiels chimiques des atomes et ions atomiques et des molécules sont calculés comme indiqués dans [12]. Dans cette relation, les températures et la pression seront fixées, les potentiels chimiques sont déterminés, seules les $N$ concentrations $n_{j}$ des espèces chimiques $j$ sont inconnues.

Nous supposons que les niveaux quantiques d'excitation électronique pour les atomes ions et molécules et les niveaux quantiques de rotation et de vibration sont peuplés suivants une distribution de Boltzmann. Ainsi, nous pouvons définir plusieurs températures, qui permettent de satisfaire la distribution de Boltzmann sur ces niveaux quantiques: la température d'excitation électronique que nous supposons identique pour les atomes, les ions et les molécules $\left(\mathrm{N}, \mathrm{O}, \mathrm{N}^{+}, \mathrm{O}^{+}, \mathrm{N}_{2}, \mathrm{O}_{2}, \mathrm{~N}_{2}{ }^{+}, \mathrm{O}_{2}{ }^{+}\right)$, la température de rotation et la température de vibration pour les molécules ionisées ou non $\left(\mathrm{N}_{2}, \mathrm{O}_{2}, \mathrm{~N}_{2}{ }^{+}, \mathrm{O}_{2}{ }^{+}\right)$. Pour les degrés de translation, nous supposons que le mouvement des atomes, des molécules et des ions suivent une distribution de Maxwell-Boltzmann à la même température de translation $T_{l}$ et que le mouvement des électrons suit une distribution de Maxwell-Boltzmann à la température de translation $T_{e}$.

La température de rotation est habituellement supposée proche de celle de la température de translation des espèces lourdes (atomes molécule, ions) $T_{l}$ alors que la température d'excitation $T_{e x}$ est supposée être proche de celle des électrons. De plus, des mesures effectuées dans plusieurs configurations [13-15] montrent que la température de vibration $T_{\text {vib }}$ est intermédiaire entre la température des espèces lourdes $T_{l}$ et la température électronique $T_{e}$. Ces conditions permettent de faire l'hypothèse simplificatrice suivante :

$$
\left\{\begin{array}{c}
T_{l}=T_{\text {rot }} \\
T_{e}=T_{e x}=\theta T_{l} \\
T_{v i b}=\sqrt{\theta} T_{l}
\end{array}\right.
$$

Nous pouvons alors définir l'équilibre thermique comme étant l'état pour lequel toutes les températures sont identiques. Il est à noter que la température de vibration choisie influence les valeurs des concentrations chimiques à l'équilibre chimique au travers du potentiel chimique et $\mu_{j}^{0}\left(T_{\text {rot }}, T_{e x}, T_{\text {vib }}\right)$ pour les molécules [16]. Cependant compte tenu que les taux de réaction sont souvent mal connus, l'hypothèse (5) sur la température de vibration est un bon compromis pour prendre en compte les résultats expérimentaux [13-15]. 
Dans cet article, nous souhaitons étudier quatre cas représentatifs : deux pressions différentes et deux états d'équilibre thermiques différents. Pour les pressions, nous choisissons, la première au niveau du sol à la pression atmosphérique et la seconde la pression de l'atmosphère aux environs de $10 \mathrm{~km}$ soit $26442 \mathrm{~Pa}$.

De plus des mesures de champ électrique sur le mont Hermon en Israël [17] et dans l'atmosphère à plusieurs altitudes [18], nous permettent d'estimer la variation du champ électrique sur une gamme de $20 \mathrm{kV} / \mathrm{m}$ à $120 \mathrm{kV} / \mathrm{m}$. De surcroit, un bilan d'énergie prenant en compte l'accélération des électrons dans le champ électrique, nous permet d'évaluer le déséquilibre thermique à $\theta \approx 2$ à 3 [19]. Les estimations des pics de températures maximales montrent des températures d'excitation de l'ordre de $T_{e x} \approx 20000 K$ [20]. Nous prenons une température d'excitation intermédiaire $T_{e x} \approx 10000 \mathrm{~K}$ et compte tenu de l'estimation du déséquilibre thermique obtenu à partir du champ électrique, nous obtenons une température des lourds de l'ordre de $T_{l} \approx 5000 \mathrm{~K}$. L'intensité du champ électrique variant durant la décharge de foudre, le plasma peut passer d'un état proche de l'équilibre thermique vers un état de déséquilibre thermique.

Afin de mettre en exergue, l'influence de l'état thermodynamique du plasma, qui est évolutif dans le temps, nous choisissons ainsi l'équilibre thermique et un état de déséquilibre thermique caractérisé par un ratio $\theta=\frac{T_{e}}{T_{l}}$ égale à 2 et la température des espèces lourdes $T_{l}$ fixée à $5000 \mathrm{~K}$.

Afin d'obtenir le minimum de la fonction d'état $G$ (4) à pression et températures fixées dont la condition nécessaire mais pas suffisante est $d G=0$, nous prenons en compte-deux contraintes supplémentaires. La première concerne la neutralité électrique du milieu :

$$
\sum_{j=1}^{N} n_{j} z_{j}=0
$$

Avec $z_{j}$ la charge de l'espèce chimique $j\left(z_{j}=0\right.$ pour $\mathrm{N}, \mathrm{O}, \mathrm{N}_{2}, \mathrm{O}_{2} ; z_{j}=1$ pour $\mathrm{N}^{+}, \mathrm{O}^{+}, \mathrm{N}_{2}{ }^{+}$, $\mathrm{O}_{2}{ }^{+}$et $z_{j}=-1$ pour les électrons).

Et la seconde est la loi de Dalton, $p_{\text {Dalton }}$, corrigée de l'abaissement de pression résultant de l'attraction coulombienne dans le mélange :

$$
p=p_{\text {Dalton }}+\Delta p=\sum_{j=1}^{N} n_{j} k T_{i}+\Delta p
$$

La correction de pression s'écrit :

$$
\Delta p=-\frac{1}{24 \pi \varepsilon_{0} \lambda_{d}{ }^{3}}
$$

avec $\lambda_{d}$ la longueur de Debye calculée en prenant en compte uniquement les électrons [21].

À la pression atmosphérique et à l'équilibre thermique, on obtient une correction de - 6,85 $10^{-5} \mathrm{~Pa}$; à la pression atmosphérique et hors de l'équilibre thermique on obtient une correction de $-5,410^{-3} \mathrm{~Pa}$, à la pression de $26442 \mathrm{~Pa}$ et à l'équilibre thermique on obtient une correction de $-1,210^{-5} \mathrm{~Pa}$ et finalement à la pression de $26442 \mathrm{~Pa}$ et hors de l'équilibre thermique on obtient une correction de $1,910^{-3} \mathrm{~Pa}$. Cette correction sur la pression est donc négligeable dans les conditions de pression et de températures que nous étudions. 
Le système d'équations (4), (6), (7) est complet, nous pouvons obtenir l'ensemble des concentrations des espèces chimiques, $n_{j} j \in[1 . . N]$, dans les quatre conditions que nous considérons. Les taux de réaction directes sont obtenus à partir des tables de C. Park et al [22], nous les prenons à la température électronique $T_{e}$ lorsque dans les réactifs les électrons sont présents et à la température des espèces lourdes $T_{l}$ lorsqu'ils ne sont pas présents. Connaissant, le taux de réaction directe, on obtient les taux de réaction inverse $k_{i}$ avec la relation (3). Les taux inverses sont alors obtenus dans l'hypothèse de l'équilibre chimique à l'équilibre thermique $\left(T_{e}=T_{l}\right)$ ou hors de l'équilibre thermique $\left(T_{e} \neq T_{l}\right)$. De surcroit, nous sélectionnons les réactions chimiques en fonctions des espèces chimiques présentes à l'équilibre chimique. Ainsi, le nombre de réactions $N_{r}$ prise en compte dépend de l'état thermodynamique et de la pression du plasma. Nous donnons les résultats ainsi que les réactions chimiques prises en compte pour les conditions de pression et de températures considérées dans le Tableau 1. L'équation d'évolution de la concentration $n_{j}$ est donnée par la relation suivante :

$$
\frac{d n_{j}}{d t}=\sum_{l=1}^{N_{r}}\left(v_{d j}^{l}-v_{i j}^{l}\right) \frac{d \xi_{l}}{d t}
$$

Nous obtenons $N$ équations différentielles que nous résolvons avec une méthode numérique de Runge-Kutta d'ordre 4 [23]. Nous prenons une concentration initiale de $79 \%$ de diazote et de $21 \%$ de dioxygène en pourcentage molaire. Nous présentons l'évolution des concentrations chimiques en fonction du temps pour les quatre cas de pression et de déséquilibre thermique, considérés, dans le Tableau 1. Nous commenterons plus en détails les résultats dans le $§ 1.3$, cependant nous pouvons noter que l'équilibre chimique, c'est-à-dire le moment à partir duquel les concentrations des espèces chimiques sont stables dans le temps, est atteint plus rapidement si le déséquilibre thermique est présent ou que la pression est plus importante. Ainsi, l'équilibre chimique est atteint plus rapidement dans le cas de la pression atmosphérique et d'un déséquilibre thermique.

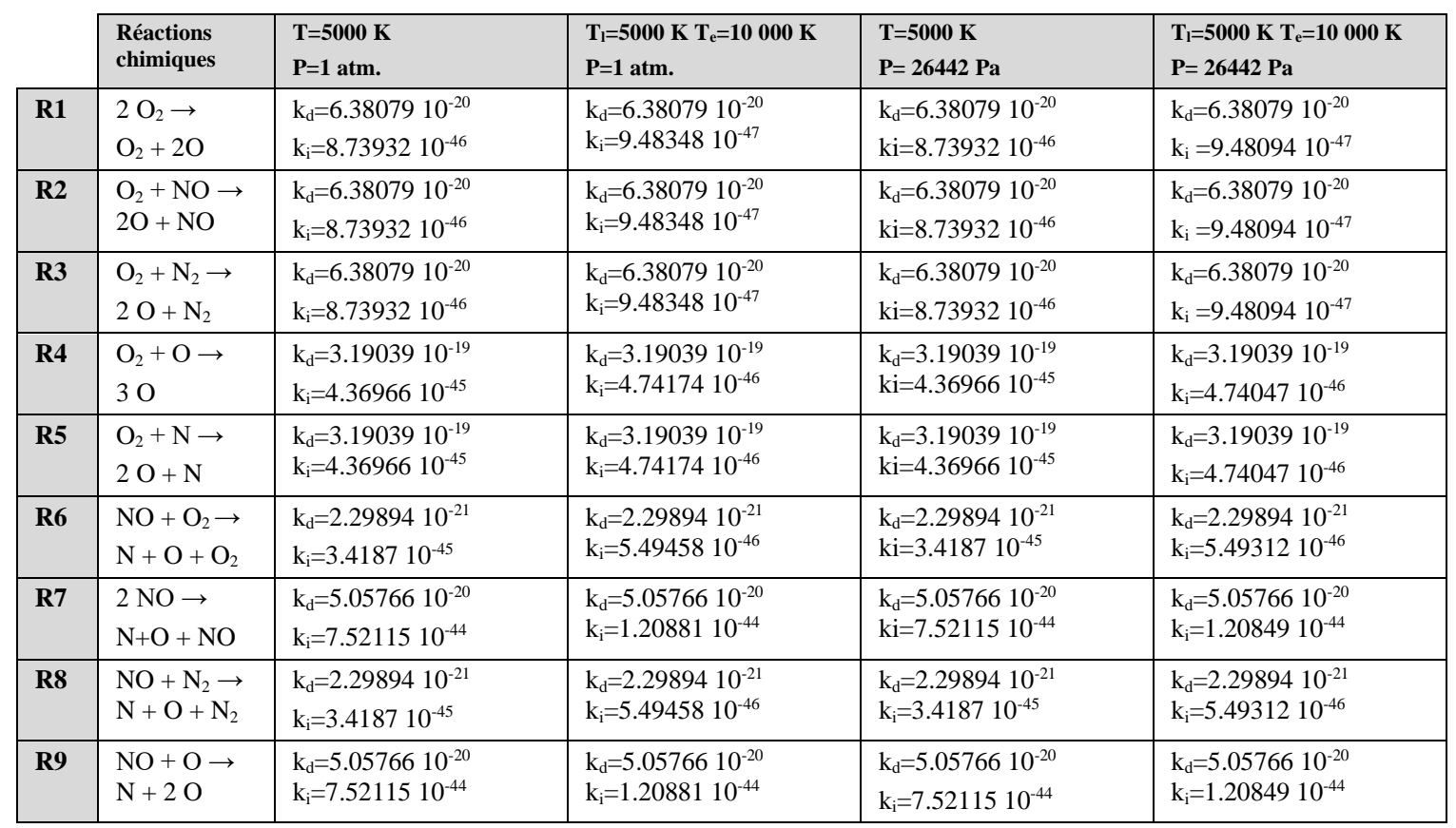




\begin{tabular}{|c|c|c|c|c|c|}
\hline R10 & $\begin{array}{l}\mathrm{NO}+\mathrm{N} \rightarrow \\
2 \mathrm{~N}+\mathrm{O}\end{array}$ & $\begin{array}{l}\mathrm{k}_{\mathrm{d}}=5.0576610^{-20} \\
\mathrm{k}_{\mathrm{i}}=7.5211510^{-44}\end{array}$ & $\begin{array}{l}\mathrm{k}_{\mathrm{d}}=5.0576610^{-20} \\
\mathrm{k}_{\mathrm{i}}=1.2088110^{-44}\end{array}$ & $\begin{array}{l}\mathrm{k}_{\mathrm{d}}=5.0576610^{-20} \\
\mathrm{k}_{\mathrm{i}}=7.5211510^{-44}\end{array}$ & $\begin{array}{l}\mathrm{k}_{\mathrm{d}}=5.0576610^{-20} \\
\mathrm{k}_{\mathrm{i}}=1.2084910^{-44}\end{array}$ \\
\hline R11 & $\begin{array}{l}\mathrm{N}_{2}+\mathrm{O}_{2} \rightarrow \\
2 \mathrm{~N}+\mathrm{O}_{2}\end{array}$ & $\begin{array}{l}\mathrm{k}_{\mathrm{d}}=2.0640610^{-24} \\
\mathrm{k}_{\mathrm{i}}=1.28610^{-45}\end{array}$ & $\begin{array}{l}\mathrm{k}_{\mathrm{d}}=2.0640610^{-24} \\
\mathrm{k}_{\mathrm{i}}=8.3617810^{-47}\end{array}$ & $\begin{array}{l}\mathrm{k}_{\mathrm{d}}=2.0640610^{-24} \\
\mathrm{k}_{\mathrm{i}}=1.28610^{-45}\end{array}$ & $\begin{array}{l}\mathrm{k}_{\mathrm{d}}=2.0640610^{-24} \\
\mathrm{k}_{\mathrm{i}}=8.3595510^{-47}\end{array}$ \\
\hline R12 & $\begin{array}{l}\mathrm{N}_{2}+\mathrm{NO} \rightarrow \\
2 \mathrm{~N}+\mathrm{NO}\end{array}$ & $\begin{array}{l}\mathrm{k}_{\mathrm{d}}=2.0640610^{-24} \\
\mathrm{k}_{\mathrm{i}}=1.28610^{-45}\end{array}$ & $\begin{array}{l}\mathrm{k}_{\mathrm{d}}=2.0640610^{-24} \\
\mathrm{k}_{\mathrm{i}}=8.3617810^{-47}\end{array}$ & $\begin{array}{l}\mathrm{k}_{\mathrm{d}}=2.0640610^{-24} \\
\mathrm{k}_{\mathrm{i}}=1.28610^{-45}\end{array}$ & $\begin{array}{l}\mathrm{k}_{\mathrm{d}}=2.0640610^{-24} \\
\mathrm{k}_{\mathrm{i}}=8.3595510^{-47}\end{array}$ \\
\hline R13 & $\begin{array}{l}2 \mathrm{~N}_{2} \rightarrow \\
2 \mathrm{~N}+\mathrm{N}_{2}\end{array}$ & $\begin{array}{l}\mathrm{k}_{\mathrm{d}}=2.0640610^{-24} \\
\mathrm{k}_{\mathrm{i}}=1.28610^{-45}\end{array}$ & $\begin{array}{l}\mathrm{k}_{\mathrm{d}}=2.0640610^{-24} \\
\mathrm{k}_{\mathrm{i}}=8.3617810^{-47}\end{array}$ & $\begin{array}{l}\mathrm{k}_{\mathrm{d}}=2.0640610^{-24} \\
\mathrm{k}_{\mathrm{i}}=1.28610^{-45}\end{array}$ & $\begin{array}{l}\mathrm{k}_{\mathrm{d}}=2.0640610^{-24} \\
\mathrm{k}_{\mathrm{i}}=8.3595510^{-47}\end{array}$ \\
\hline R14 & $\begin{array}{l}\mathrm{N}_{2}+\mathrm{O} \rightarrow \\
2 \mathrm{~N}+\mathrm{O}\end{array}$ & $\begin{array}{l}\mathrm{k}_{\mathrm{d}}=8.84597 \quad 10^{-24} \\
\mathrm{k}_{\mathrm{i}}=5.51143 \quad 10^{-45}\end{array}$ & $\begin{array}{l}\mathrm{k}_{\mathrm{d}}=8.84597 \quad 10^{-24} \\
\mathrm{k}_{\mathrm{i}}=3.58362 \quad 10^{-46}\end{array}$ & $\begin{array}{l}\mathrm{k}_{\mathrm{d}}=8.8459710^{-24} \\
\mathrm{k}_{\mathrm{i}}=5.51143 \quad 10^{-45}\end{array}$ & $\begin{array}{l}\mathrm{k}_{\mathrm{d}}=8.84597 \quad 10^{-24} \\
\mathrm{k}_{\mathrm{i}}=3.58266 \quad 10^{-46}\end{array}$ \\
\hline R15 & $\begin{array}{l}\mathrm{N}_{2}+\mathrm{N} \rightarrow \\
3 \mathrm{~N}\end{array}$ & $\begin{array}{l}\mathrm{k}_{\mathrm{d}}=8.8459710^{-24} \\
\mathrm{k}_{\mathrm{i}}=5.5114310^{-45}\end{array}$ & $\begin{array}{l}\mathrm{k}_{\mathrm{d}}=8.84597 \quad 10^{-24} \\
\mathrm{k}_{\mathrm{i}}=3.5836210^{-46}\end{array}$ & $\begin{array}{l}\mathrm{k}_{\mathrm{d}}=8.84597 \quad 10^{-24} \\
\mathrm{k}_{\mathrm{i}}=5.51143 \quad 10^{-45}\end{array}$ & $\begin{array}{l}\mathrm{k}_{\mathrm{d}}=8.8459710^{-24} \\
\mathrm{k}_{\mathrm{i}}=3.5826610^{-46}\end{array}$ \\
\hline R16 & $\begin{array}{l}\mathrm{N}_{2}+\mathrm{e}^{-} \rightarrow \\
2 \mathrm{~N}+\mathrm{e}^{-}\end{array}$ & $\begin{array}{l}\mathrm{k}_{\mathrm{d}}=8.8459710^{-22} \\
\mathrm{k}_{\mathrm{i}}=5.5114310^{-43}\end{array}$ & $\begin{array}{l}\mathrm{k}_{\mathrm{d}}=8.8459710^{-24} \\
\mathrm{k}_{\mathrm{i}}=3.5836210^{-46}\end{array}$ & $\begin{array}{l}\mathrm{k}_{\mathrm{d}}=8.84597 \quad 10^{-24} \\
\mathrm{k}_{\mathrm{i}}=5.51143 \quad 10^{-45}\end{array}$ & $\begin{array}{l}\mathrm{k}_{\mathrm{d}}=8.8459710^{-24} \\
\mathrm{k}_{\mathrm{i}}=3.5826610^{-46}\end{array}$ \\
\hline R17 & $\begin{array}{l}\mathrm{N}_{2}+\mathrm{O} \rightarrow \\
\mathrm{NO}+\mathrm{N}\end{array}$ & $\begin{array}{l}\mathrm{k}_{\mathrm{d}}=9.8227210^{-20} \\
\mathrm{k}_{\mathrm{i}}=4.1154410^{-17}\end{array}$ & $\begin{array}{l}\mathrm{k}_{\mathrm{d}}=9.8227210^{-20} \\
\mathrm{k}_{\mathrm{i}}=1.6649510^{-17}\end{array}$ & $\begin{array}{l}\mathrm{k}_{\mathrm{d}}=9.8227210^{-20} \\
\mathrm{k}_{\mathrm{i}}=4.1154410^{-17}\end{array}$ & $\begin{array}{l}\mathrm{k}_{\mathrm{d}}=9.8227210^{-20} \\
\mathrm{k}_{\mathrm{i}}=1.6649510^{-17}\end{array}$ \\
\hline R18 & $\begin{array}{l}\mathrm{NO}+\mathrm{O} \rightarrow \\
\mathrm{O}_{2}+\mathrm{N}\end{array}$ & $\begin{array}{l}\mathrm{k}_{\mathrm{d}}=2.8815110^{-19} \\
\mathrm{k}_{\mathrm{i}}=3.1286110^{-17}\end{array}$ & $\begin{array}{l}\mathrm{k}_{\mathrm{d}}=2.8815110^{-19} \\
\mathrm{k}_{\mathrm{i}}=4.6337710^{-17}\end{array}$ & $\begin{array}{l}\mathrm{k}_{\mathrm{d}}=2.88151 \quad 10^{-19} \\
\mathrm{k}_{\mathrm{d}}=3.12861 \quad 10^{-17}\end{array}$ & $\begin{array}{l}\mathrm{k}_{\mathrm{d}}=2.8815110^{-19} \\
\mathrm{k}_{\mathrm{i}}=4.6337710^{-17}\end{array}$ \\
\hline R19 & $\begin{array}{l}\mathrm{N}+\mathrm{O} \rightarrow \\
\mathrm{NO}^{+}+\mathrm{e}^{-}\end{array}$ & $\begin{array}{l}\mathrm{k}_{\mathrm{d}}=2.9847710^{-24} \\
\mathrm{k}_{\mathrm{i}}=1.4129510^{-17}\end{array}$ & $\begin{array}{l}\mathrm{k}_{\mathrm{d}}=2.9847710^{-24} \\
\mathrm{k}_{\mathrm{i}}=2.1195510^{-22}\end{array}$ & $\begin{array}{l}\mathrm{k}_{\mathrm{d}}=2.9847710^{-24} \\
\mathrm{k}_{\mathrm{d}}=1.4129510^{-17}\end{array}$ & $\begin{array}{l}\mathrm{k}_{\mathrm{d}}=2.9847710^{-24} \\
\mathrm{k}_{\mathrm{i}}=1.0196110^{-22}\end{array}$ \\
\hline $\mathbf{R 2 0}$ & $\begin{array}{l}2 \mathrm{~N} \rightarrow \\
\mathrm{N}_{2}^{+}+\mathrm{e}^{-} \\
\end{array}$ & & $\begin{array}{l}\mathrm{k}_{\mathrm{d}}=1.8374910^{-20} \\
\mathrm{k}_{\mathrm{i}}=6.194110^{-17}\end{array}$ & & $\begin{array}{l}\mathrm{k}_{\mathrm{d}}=1.8374910^{-20} \\
\mathrm{k}_{\mathrm{i}}=2.9796510^{-17}\end{array}$ \\
\hline $\mathbf{R} 21$ & $\begin{array}{l}2 \mathrm{O} \rightarrow \\
\mathrm{O}_{2}^{+}+\mathrm{e}^{-}\end{array}$ & & $\begin{array}{l}\mathrm{k}_{\mathrm{d}}=1.8237710^{-24} \\
\mathrm{k}_{\mathrm{i}}=7.6445710^{-19}\end{array}$ & & $\begin{array}{l}\mathrm{k}_{\mathrm{d}}=1.8237710^{-24} \\
\mathrm{k}_{\mathrm{i}}=3.677410^{-19}\end{array}$ \\
\hline R22 & $\begin{array}{l}\mathrm{O}+\mathrm{e}^{-} \rightarrow \\
\mathrm{O}^{+}+2 \mathrm{e}^{-}\end{array}$ & & $\begin{array}{l}\mathrm{k}_{\mathrm{d}}=6.7548410^{-19} \\
\mathrm{k}_{\mathrm{i}}=5.604910^{-38}\end{array}$ & & $\begin{array}{l}\mathrm{k}_{\mathrm{d}}=6.7548410^{-19} \\
\mathrm{k}_{\mathrm{i}}=2.695510^{-38}\end{array}$ \\
\hline $\mathbf{R} 23$ & $\begin{array}{l}\mathrm{N}+\mathrm{e}^{-} \rightarrow \\
\mathrm{N}^{+}+2 \mathrm{e}^{-}\end{array}$ & & $\begin{array}{l}\mathrm{k}_{\mathrm{d}}=1.0802210^{-19} \\
\mathrm{k}_{\mathrm{i}}=3.9130910^{-39}\end{array}$ & & $\begin{array}{l}\mathrm{k}_{\mathrm{d}}=1.0802210^{-19} \\
\mathrm{k}_{\mathrm{i}}=1.88188 \quad 10^{-39}\end{array}$ \\
\hline $\mathbf{R} 24$ & $\begin{array}{l}\mathrm{NO}^{+}+\mathrm{O} \rightarrow \\
\mathrm{N}^{+}+\mathrm{O}_{2}\end{array}$ & & $\begin{array}{l}\mathrm{k}_{\mathrm{d}}=2.314110^{-23} \\
\mathrm{k}_{\mathrm{i}}=7.94263 \quad 10^{-18}\end{array}$ & & $\begin{array}{l}\mathrm{k}_{\mathrm{d}}=2.314110^{-23} \\
\mathrm{k}_{\mathrm{i}}=7.9426310^{-18}\end{array}$ \\
\hline R25 & $\begin{array}{l}\mathrm{O}_{2}^{+}+\mathrm{N} \rightarrow \\
\mathrm{N}^{+}+\mathrm{O}_{2}\end{array}$ & & $\begin{array}{l}\mathrm{k}_{\mathrm{d}}=1.5617610^{-18} \\
\mathrm{k}_{\mathrm{i}}=9.0812710^{-17}\end{array}$ & & $\begin{array}{l}\mathrm{k}_{\mathrm{d}}=1.5617610^{-18} \\
\mathrm{k}_{\mathrm{i}}=9.0812710^{-17}\end{array}$ \\
\hline R26 & $\begin{array}{l}\mathrm{NO}+\mathrm{O}^{+} \rightarrow \\
\mathrm{N}^{+}+\mathrm{O}_{2}\end{array}$ & & $\begin{array}{l}\mathrm{k}_{\mathrm{d}}=1.1631410^{-19} \\
\mathrm{k}_{\mathrm{i}}=8.16593 \quad 10^{-18}\end{array}$ & & $\begin{array}{l}\mathrm{k}_{\mathrm{d}}=1.16314 \quad 10^{-19} \\
\mathrm{k}_{\mathrm{i}}=8.16593 \quad 10^{-18}\end{array}$ \\
\hline R27 & $\begin{array}{l}\mathrm{O}_{2}^{+}+\mathrm{N}_{2} \rightarrow \\
\mathrm{N}_{2}^{+}+\mathrm{O}_{2}\end{array}$ & & $\begin{array}{l}\mathrm{k}_{\mathrm{d}}=4.7960310^{-21} \\
\mathrm{k}_{\mathrm{i}}=1.0513210^{-18}\end{array}$ & & $\begin{array}{l}\mathrm{k}_{\mathrm{d}}=4.7960310^{-21} \\
\mathrm{k}_{\mathrm{i}}=1.0513210^{-18}\end{array}$ \\
\hline R28 & $\begin{array}{l}\mathrm{NO}^{+}+\mathrm{N} \rightarrow \\
\mathrm{O}^{+}+\mathrm{N}_{2} \\
\end{array}$ & & $\begin{array}{l}\mathrm{k}_{\mathrm{d}}=4.4177510^{-22} \\
\mathrm{k}_{\mathrm{i}}=1.27422 \quad 10^{-20}\end{array}$ & & $\begin{array}{l}\mathrm{k}_{\mathrm{d}}=4.4177510^{-22} \\
\mathrm{k}_{\mathrm{i}}=1.2742210^{-20}\end{array}$ \\
\hline $\mathbf{R} 29$ & $\begin{array}{l}\mathrm{NO}^{+}+\mathrm{O}_{2} \rightarrow \\
\mathrm{O}_{2}^{+}+\mathrm{NO}\end{array}$ & & $\begin{array}{l}\mathrm{k}_{\mathrm{d}}=1.9301610^{-18} \\
\mathrm{k}_{\mathrm{i}}=7.0848110^{-17}\end{array}$ & & $\begin{array}{l}\mathrm{k}_{\mathrm{d}}=1.9301610^{-18} \\
\mathrm{k}_{\mathrm{i}}=7.0848110^{-17}\end{array}$ \\
\hline $\mathbf{R 3 0}$ & $\begin{array}{l}\mathrm{NO}^{+}+\mathrm{O} \rightarrow \\
\mathrm{O}_{2}^{+}+\mathrm{N}\end{array}$ & & $\begin{array}{l}\mathrm{k}_{\mathrm{d}}=8.4936610^{-21} \\
\mathrm{k}_{\mathrm{i}}=5.0135510^{-17}\end{array}$ & & $\begin{array}{l}\mathrm{k}_{\mathrm{d}}=8.4936610^{-21} \\
\mathrm{k}_{\mathrm{i}}=5.0135510^{-17}\end{array}$ \\
\hline R31 & $\begin{array}{l}\mathrm{O}^{+}+\mathrm{N}_{2} \rightarrow \\
\mathrm{N}_{2}^{+}+\mathrm{O}\end{array}$ & & $\begin{array}{l}\mathrm{k}_{\mathrm{d}}=3.3565410^{-19} \\
\mathrm{k}_{\mathrm{i}}=5.524210^{-19}\end{array}$ & & $\begin{array}{l}\mathrm{k}_{\mathrm{d}}=3.3565410^{-19} \\
\mathrm{k}_{\mathrm{i}}=5.524210^{-19}\end{array}$ \\
\hline R32 & $\begin{array}{l}\mathrm{NO}^{+}+\mathrm{N} \rightarrow \\
\mathrm{N}_{2}^{+}+\mathrm{O}\end{array}$ & & $\begin{array}{l}\mathrm{k}_{\mathrm{d}}=9.8683610^{-20} \\
\mathrm{k}_{\mathrm{i}}=4.6845110^{-18}\end{array}$ & & $\begin{array}{l}\mathrm{k}_{\mathrm{d}}=9.8683610^{-20} \\
\mathrm{k}_{\mathrm{i}}=4.6845110^{-18}\end{array}$ \\
\hline R33 & $\begin{array}{l}\mathrm{O}+\mathrm{N}_{2} \mathrm{O} \rightarrow \\
2 \mathrm{NO}\end{array}$ & $\begin{array}{l}\mathrm{k}_{\mathrm{d}}=9.997910^{-18} \\
\mathrm{k}_{\mathrm{i}}=9.1371510^{-21}\end{array}$ & & & \\
\hline R34 & $\begin{array}{l}\mathrm{N}_{2} \mathrm{O}+\mathrm{O} \rightarrow \\
\mathrm{O}_{2}+\mathrm{N}_{2}\end{array}$ & $\begin{array}{l}\mathrm{k}_{\mathrm{d}}=8.4710410^{-18} \\
\mathrm{k}_{\mathrm{i}}=2.0062510^{-21}\end{array}$ & & & \\
\hline R35 & $\begin{array}{l}\mathrm{N}+\mathrm{NO} \rightarrow \\
\mathrm{N}_{2} \mathrm{O}\end{array}$ & $\begin{array}{l}\mathrm{k}_{\mathrm{d}}=1.7811210^{-17} \\
\mathrm{k}_{\mathrm{i}}=1.3105610^{10}\end{array}$ & & & \\
\hline
\end{tabular}

Tableau 1. Taux de réaction directes $k_{d}$ et inverses $k_{i}$ calculés pour les quatre conditions initiales. 


\subsection{Calcul des permittivités}

Si on néglige les dipôles créés par l'onde électromagnétique incidente, en supposant que le plasma est non-magnétique et qu'aucune zone de charge n'est créée, on peut écrire à partir de la loi de Newton et de l'équation de Maxwell-Ampère [9, 24]:

$$
\varepsilon=\varepsilon_{0}\left(1-\frac{\omega_{p}^{2}}{\omega\left(\omega-\mathrm{i} v_{e_{P}}\right)}\right)
$$

Que nous pouvons écrire sous la forme de deux permittivités relatives l'une concerne la partie réelle $\varepsilon_{r r}$ et l'autre concerne la partie imaginaire $\varepsilon_{r i}$ :

$$
\varepsilon=\varepsilon_{0}\left(\varepsilon_{r r}+\mathrm{i} \varepsilon_{r i}\right)=\varepsilon_{0} \varepsilon_{r}
$$

Où $\varepsilon_{r r}=1-\frac{\omega_{p}^{2}}{\omega^{2}+v_{e P}^{2}}$ et $\varepsilon_{r c}=-\frac{\omega_{p}^{2} v_{e P}}{\omega\left(\omega^{2}+v_{e P}^{2}\right)}$

Avec $\omega$ la pulsation de l'onde incidente à la fréquence $f(\omega=2 \pi f), \omega_{p}$ la pulsation du plasma et $v_{e P}$ la fréquence de collision entre les électrons et les particules $P$ (ions, atomes et molécules) du plasma hormis les électrons.

La pulsation du plasma dépend de la concentration électronique $n_{e}$ :

$$
\omega_{p}=\sqrt{\frac{e^{2} n_{e}}{m_{e} \varepsilon_{0}}}
$$

Avec $e$ la charge électrique élémentaire, $m_{e}$ la masse d'un électron et $\varepsilon_{0}$ la permittivité du vide.

Plusieurs fréquences de collision peuvent être définies en fonction de la grandeur de transport à calculer [25], on parle alors de fréquence de collision dite effective. Elles peuvent être calculées en fonction des sections efficaces de transfert de quantité de mouvement [26]. Nous proposons d'obtenir la fréquence de collision en fonction des intégrales de collision $\bar{Q}_{e P}^{(1,1)}$ :

$$
\frac{v_{e P}}{n_{P}}=\bar{v}_{e} \bar{Q}_{e P}^{(1,1)}=\left(\frac{8 k T_{e}}{\pi m_{e}}\right)^{1 / 2} \bar{Q}_{e P}^{(1,1)}
$$

Avec $\bar{v}_{e}$ la vitesse moyenne des électrons suivant une distribution de Maxwell-Boltzmann.

Pour les espèces chimiques chargées électriquement, les sections efficaces de collision $\bar{Q}_{e P}^{(1,1)}$ pour les particules $P$ électriquement chargées sont obtenue en supposons que l'interaction suit un potentiel de Coulomb écranté [26, 27]. La longueur de Debye étant calculée en prenant en compte uniquement les électrons [21]. 
Pour les espèces chimiques neutres, dans la Figure 1, nous comparons nos résultats, avec ceux d'autres auteurs [26-29] soit les fréquences sont directement comparée avec celles de la littérature $[28,29]$ ou soit elles sont comparées avec celles obtenues à partir des intégrales de collision [26, 27]. Les fréquences individuelles de collisions sont celles obtenues pour les collisions:

- entre les électrons et l'oxygène monoatomique $(\mathrm{O})$,

- entre les électrons et l'azote monoatomique $(\mathrm{N})$,

- entre les électrons et le dioxygène $\left(\mathrm{O}_{2}\right)$,

- entre les électrons et l'oxygène le diazote $\left(\mathrm{N}_{2}\right)$,

- et entre les électrons et le monoxyde d'azote (NO).

a)

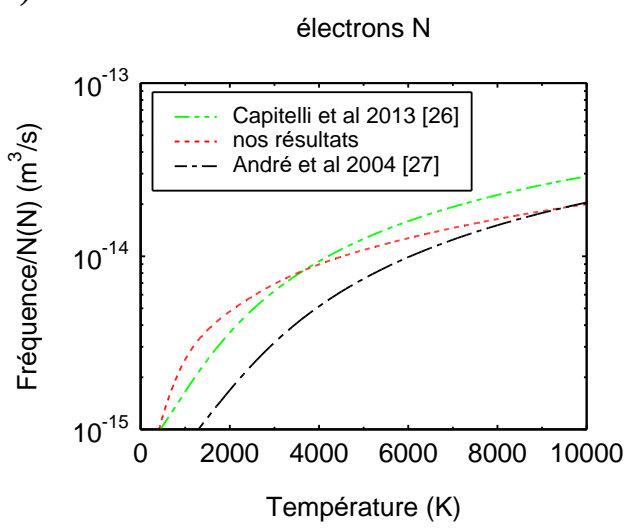

c)

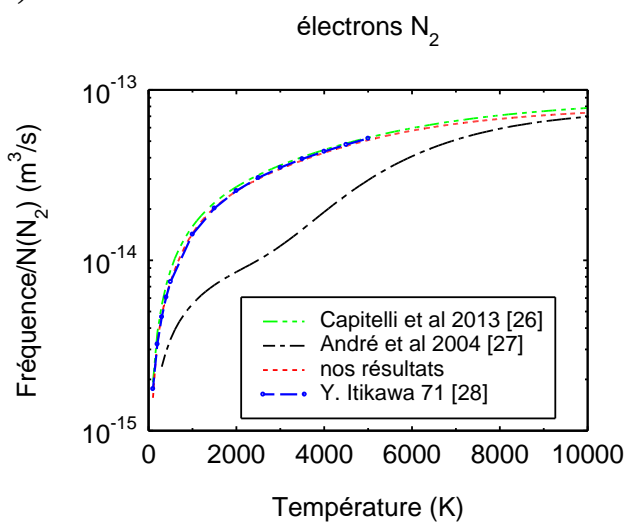

b)

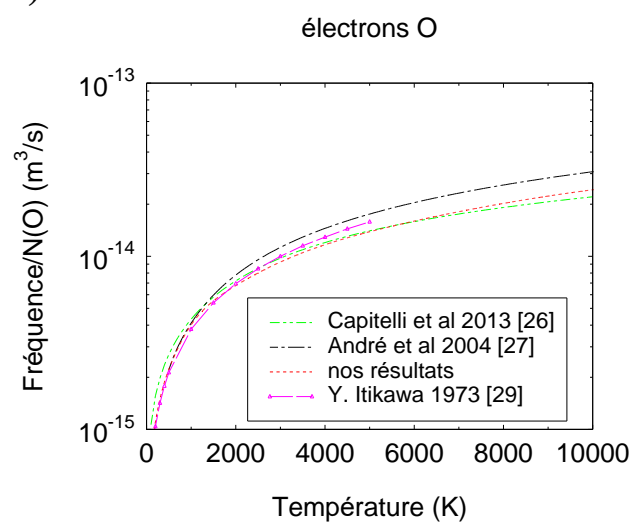

d)

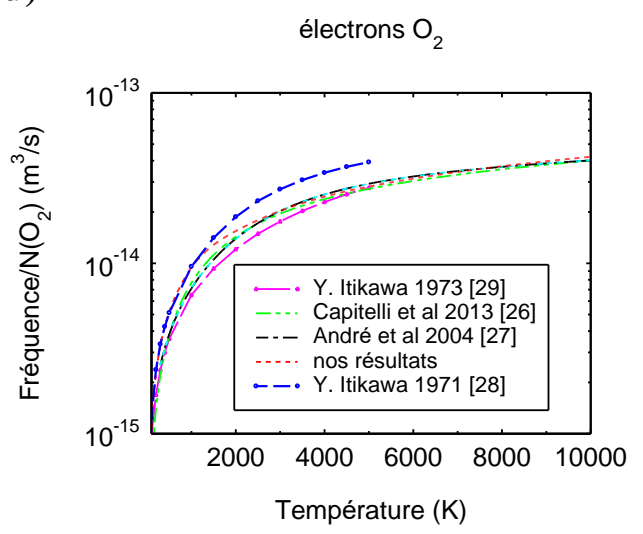

e)

électrons NO

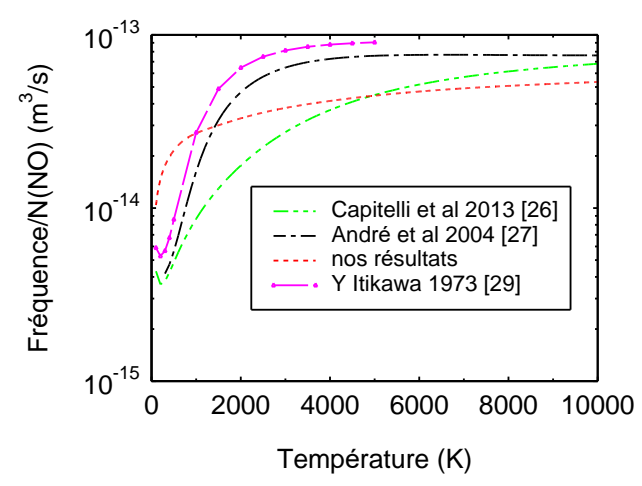

Figure 1. Fréquence de collision individuelle calculée en fonction de la température pour différente espèces chimiques : a) azote monoatomique $(\mathrm{N})$ b) oxygène monoatomique $(\mathrm{O})$ c) diazote $\left(\mathrm{N}_{2}\right)$ d) dioxygène $\left(\mathrm{O}_{2}\right)$ e) monoxyde d'azote $(\mathrm{NO})$. 
Nous remarquons que les résultats pour les collisions entre les électrons et les atomes d'oxygène $(\mathrm{O})$ et d'azote $(\mathrm{N})$ et celles entre les électrons et le dioxygène $\left(\mathrm{O}_{2}\right)$ sont similaires entre les différents auteurs [26-29].

En ce qui concerne les molécules diazote $\left(\mathrm{N}_{2}\right)$ la différence est notable entre nos calculs réalisés en 2004 [27] et les calculs des autres auteurs aux basses températures ( $<5000 \mathrm{~K})$. En 2004 [27], nous avions pris en compte les travaux de Neynaberd et al [30]. Nous les avons repris avec les travaux de Sourd et al [31]. Sur les graphes, nous présentons nos résultats avec cette mise à jour des données. Les résultats ainsi obtenus sont proches de ceux de Itikawa [28] et ceux obtenus à partir des données de Capitelli et al [26]. Cependant, nous devons noter que la différence entre les auteurs s'amenuise pour des températures supérieures à $5000 \mathrm{~K}$.

Des différences notables concernent les fréquences de collision entre les électrons et le monoxyde d'azote (NO). En 2004, nous avions pris les sections de transfert de quantité de mouvement de Spencer et al [32] que nous avons repris avec les travaux de [33, 34]. Nous présentons nos résultats avec cette mise à jour des sections de transfert de quantité de mouvement sur la figure 1.e.

Afin d'évaluer les variations de fréquence individuelle sur les résultats de permittivité, nous calculons la permittivité relative réelle $\varepsilon_{r r}$ et la permittivité relative imaginaire $\varepsilon_{r i}$ en prenant des fréquences individuelles de collision calculées avec trois sources de section efficace de collision $\bar{Q}_{e P}^{(1,1)}$ (Capitelli et al [26], André et al [27] et nos résultats résultant d'une mise à jour et d'un choix plus judicieux des sections efficaces de transfert de quantité de mouvement). Nous choisissons les concentrations d'un plasma d'air à l'équilibre thermique produit à la pression atmosphérique à $1 \mathrm{~ms}$ et à la température de $5000 \mathrm{~K}$ (Figure 3.a.). Les résultats obtenus avec différentes sources de données pour les sections efficaces de collision $\bar{Q}_{e P}^{(1,1)}$ sont présentés sur la figure 2.a. pour la permittivité relative réelle $\varepsilon_{r r}$ et sur la figure 2.b. pour la permittivité relative imaginaire $\varepsilon_{r i}$. Une grande différence existe entre nos calculs réalisés avec les sections efficaces utilisées en 2004 et les plus récentes. Ainsi, on note une différence de l'ordre de 100\% pour la permittivité relative réelle. Pour l'expliquer, nous observons que l'espèce majoritaires est le diazote $\mathrm{N}_{2}$ (Figure 3.a.). C'est donc la fréquence individuelle du diazote figure 1.c. qui influe plus particulièrement sur la permittivité réelle. Celle-ci montre une différence notable entre les sources des sections efficaces de collision. Bien que la fréquence de collision individuelle du diazote $\left(\mathrm{N}_{2}\right)$ soit proche des résultats obtenus avec les sections efficaces de Capitelli et al [26] une différence $(<10 \%)$ persiste sur la permittivité relative. En effet, puisque la concentration en monoxyde d'azote (NO) et inférieure aux concentrations de l'oxygène $(\mathrm{O})$ et l'azote monoatomique $(\mathrm{N})$, nous pourrions penser que cette interaction joue peu. Cependant au niveau des fréquences individuelles, Figure 1, on remarque qu'à $5000 \mathrm{~K}$, la fréquence individuelle du monoxyde d'azote (NO), Figure 1.e est environ 10 fois plus élevée que pour les espèces monoatomiques $\mathrm{O}$ et $\mathrm{N}$, Figures 1.a et 1.b. Ainsi, les valeurs disparates des fréquences individuelles de NO en fonctions des sources expliquent la différence $(<10 \%)$ sur la permittivité relative réelle.

La permittivité relative imaginaire est moins impactée par les fréquences de collision individuelle car elle dépend plus fortement de la pulsation de l'onde incidente $-1 / \omega^{3}$ (relation 11). 
a)

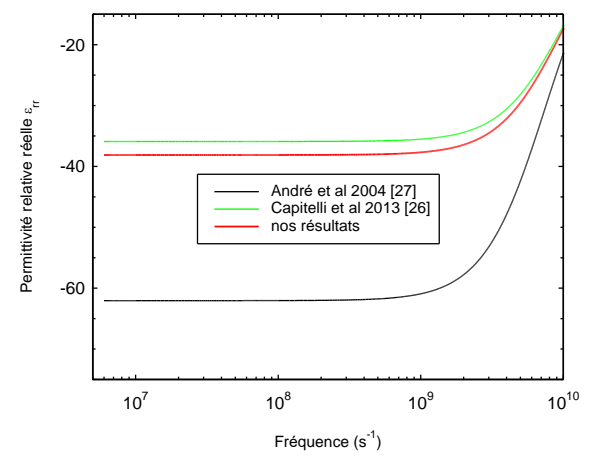

b)

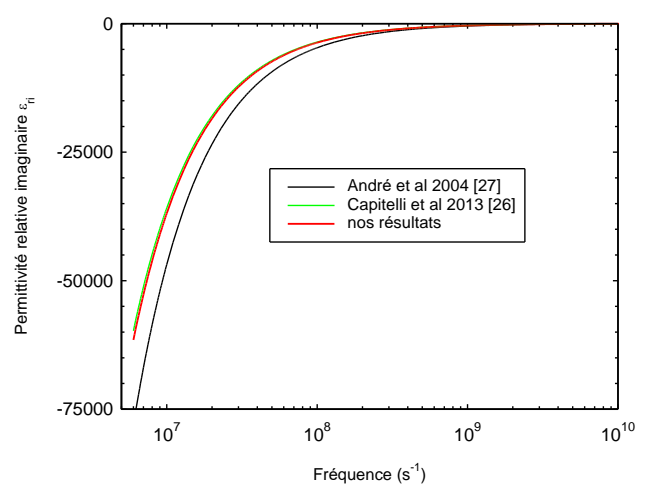

Figure 2. Évaluation de l'erreur commise en fonction des bases de données. Permittivité partie réelle a) et partie imaginaire b) d'un plasma d'air à la pression atmosphérique, à une température de $5000 \mathrm{~K}$, à l'équilibre thermique et au temps de $10^{-3} \mathrm{~s}$ en fonction de la fréquence d'excitation de l'onde incidente.

\subsection{Résultats}

Sur la Figure 3, nous avons représenté l'évolution des concentrations en fonction des conditions de pression et d'équilibre considérées. Nous notons que l'espèce majoritaire est le diazote jusqu'à obtenir l'équilibre chimique. Le dioxygène se dissocie au bout d'environ $1 \mu$ s à la pression atmosphérique (101325 Pa) et au bout d'environ $13 \mu$ s à la pression de $26442 \mathrm{~Pa}$. Durant la dissociation du dioxygène, on relève une augmentation de la production de monoxyde d'azote qui se dissocie pour atteindre son niveau d'équilibre. Lorsque le déséquilibre thermique n'est pas présent le taux d'ionisation est plus faible. En effet, lorsque la température des électrons est plus élevée, le système contient plus d'énergie dont une part sert à l'ionisation. Dans le cas de l'équilibre thermique, la neutralité électrique est réalisée entre les ions monoxyde d'azote et les électrons tout au long du processus réactif. Alors que dans le cas du déséquilibre thermique, on observe que dans un premier temps on obtient la neutralité électrique entre les ions monoatomiques oxygène $\mathrm{O}^{+}$et les électrons jusqu'à $0,25 \mu$ s à la pression atmosphérique et jusqu'à $10 \mu$ s à la pression de 26442 Pa puis la neutralité électrique est réalisée entre les ions monoxyde d'azote et les électrons.

L'équilibre chimique est atteint plus rapidement pour les espèces majoritaires en l'occurrence les espèces chimiques neutres. Les espèces chimiques ionisées mettent plus de temps à atteindre l'équilibre chimique, par exemple à la pression correspondant à $10 \mathrm{~km}$ d'altitude et à l'équilibre thermique, on obtient un temps de l'ordre de $0,7 \mathrm{~ms}$ pour les espèces chimiques neutres et $9 \mathrm{~ms}$ pour les espèces chimiques chargées (Tableau 2). Lorsque le déséquilibre thermique est présent, l'équilibre chimique est atteint plus rapidement pour les espèces chargées et plus lentement pour les espèces neutres. Par exemple, à $10 \mathrm{~km}$ d'altitude, on atteint l'équilibre chimique pour les espèces chargées en $9 \mathrm{~ms}$ à l'équilibre thermique et en $4 \mathrm{~ms}$ hors de l'équilibre et en ce qui concerne les espèces chimiques neutres en $0,7 \mathrm{~ms}$ à l'équilibre thermique et en 2,4 ms hors de l'équilibre. Lorsque la pression est plus grande, les collisions sont plus importantes on atteint l'équilibre chimique plus rapidement, c'est ce que nous observons à la fois pour les espèces chimiques neutres en passant d'un temps de $0,7 \mathrm{~ms}$ à $0,12 \mathrm{~ms}$ et pour les espèces chimiques ionisées en passant de $9 \mathrm{~ms}$ à $2,7 \mathrm{~ms}$. 
a)

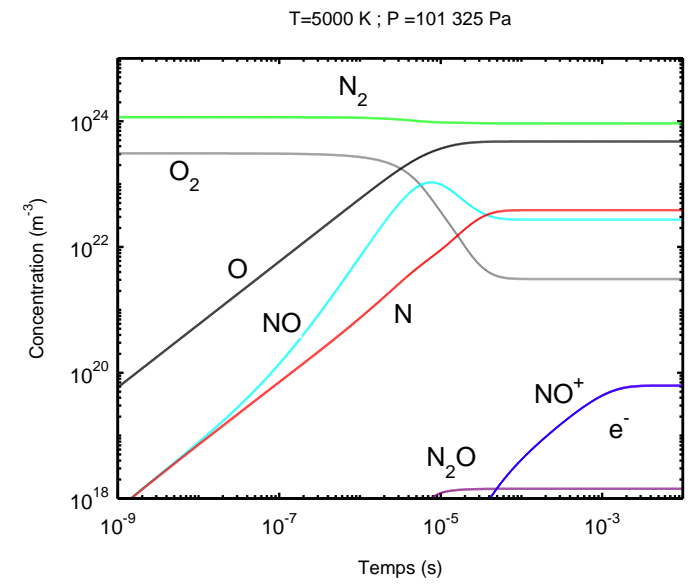

c)

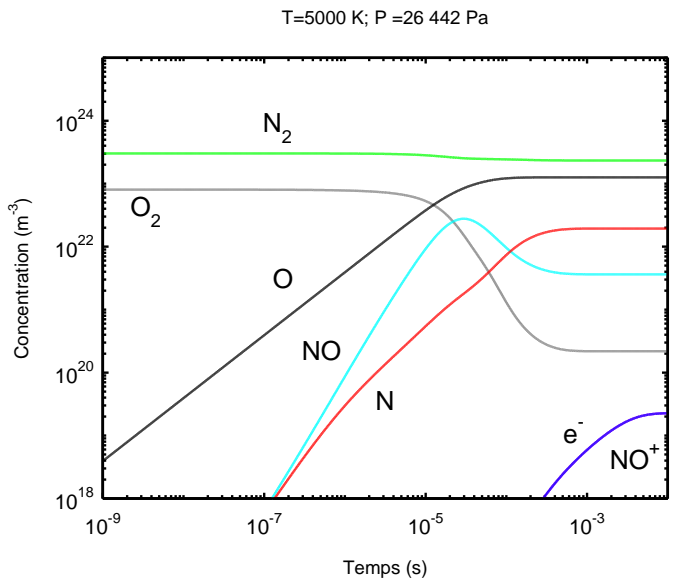

b)

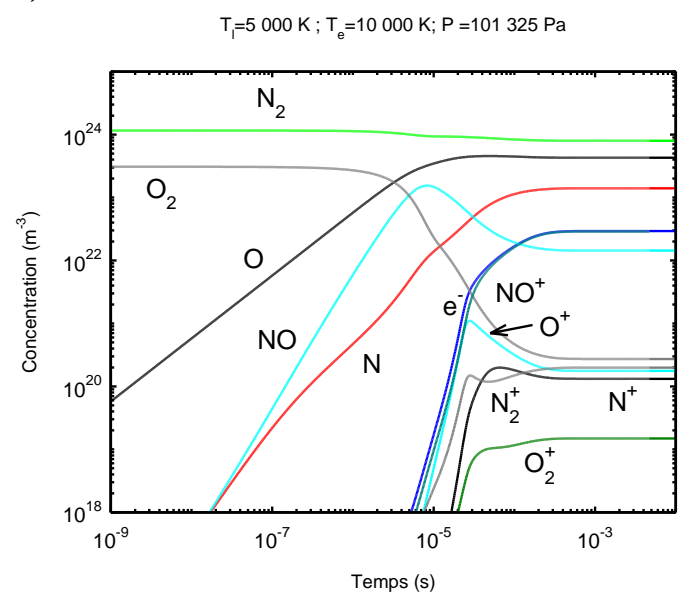

d)

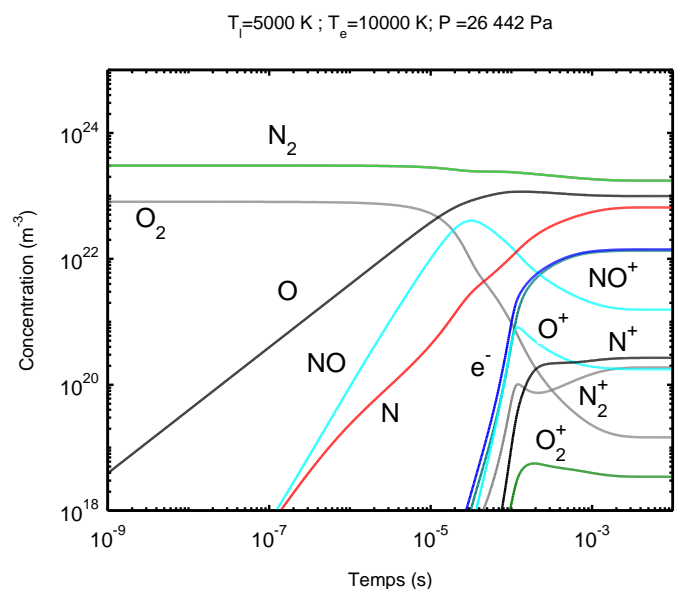

Figure 3. Évolution des concentrations en fonction du temps pour les quatre conditions considérées : a) pression atmosphérique à l'équilibre thermique ; b) pression atmosphérique hors de l'équilibre thermique ; c) pression à $10 \mathrm{~km}$ d'altitude à l'équilibre thermique ; d) pression à $10 \mathrm{~km}$ d'altitude hors de l'équilibre thermique.

\begin{tabular}{|c|c|c|c|}
\hline & Espèces chimiques & $\boldsymbol{\theta}=\mathbf{1}$ & $\boldsymbol{\theta}=\mathbf{2}$ \\
\hline $\mathbf{P}=101325 \mathbf{P a}$ & neutres majoritaires: & $0,12 \mathrm{~ms}$ & $0,3 \mathrm{~ms}$ \\
\cline { 2 - 4 } (Altitude: 0 km) & ionisées : & $2,7 \mathrm{~ms}$ & $0,5 \mathrm{~ms}$ \\
\hline $\mathbf{P = 2 6} 442 \mathbf{P a}$ & neutres majoritaires: & $0,7 \mathrm{~ms}$ & $2,4 \mathrm{~ms}$ \\
\cline { 2 - 4 } (Altitude: $10 \mathrm{~km}$ ) & ionisées : & $9 \mathrm{~ms}$ & $4 \mathrm{~ms}$ \\
\hline
\end{tabular}

Tableau 2. Temps nécessaires pour atteindre l'équilibre chimique en fonction du type d'espèces chimiques neutres ou ionisées et des conditions de pression et d'équilibre thermique.

Sur la Figure 4, nous montrons l'évolution des permittivités relatives en fonction de la pression et de l'état d'équilibre du plasma. Nous observons que lorsque la pression est plus faible, la permittivité relative réelle du plasma est plus faible. En effet, les concentrations sont plus faibles et par conséquent la fréquence de collision des électrons est plus faible. 
a.1)

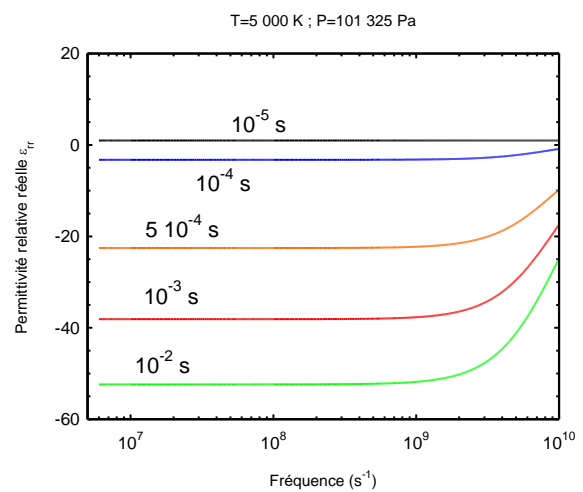

b.1)

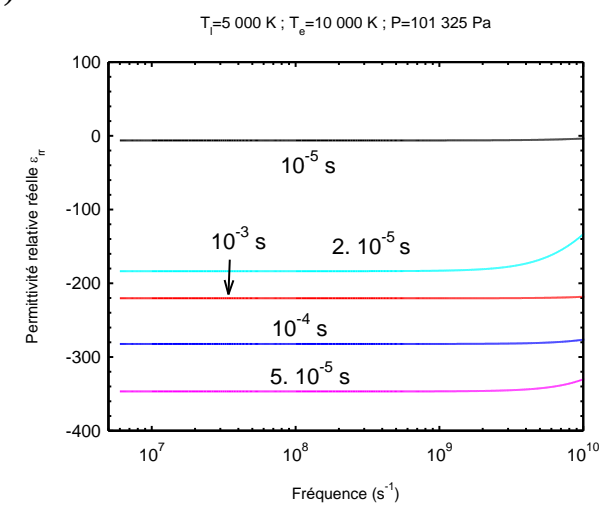

c.1)

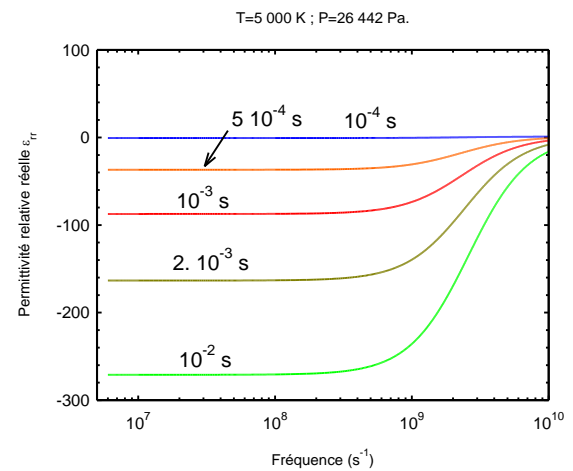

d.1)

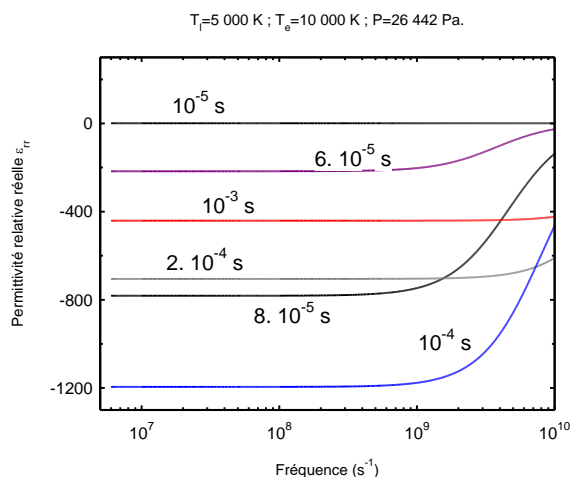

a.2)

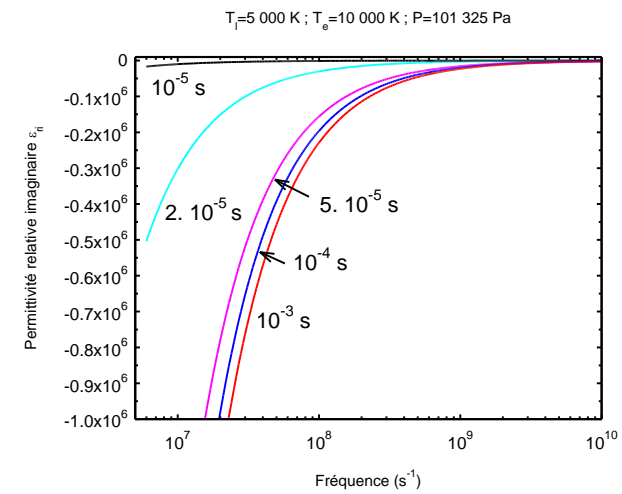

b.2)

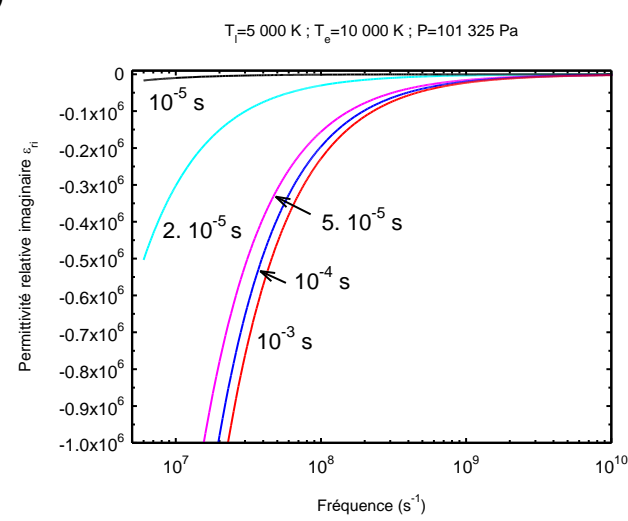

c.2)

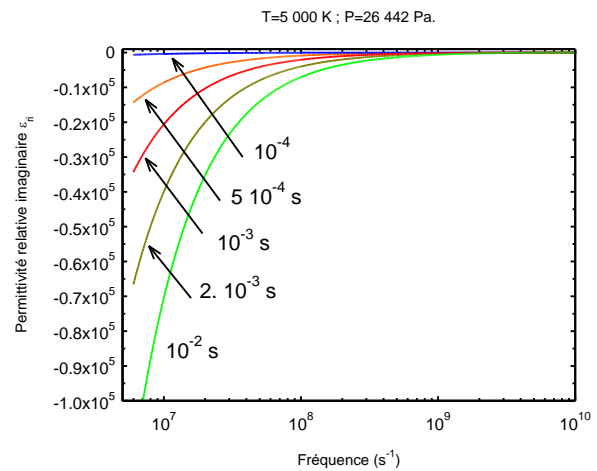

d.2)

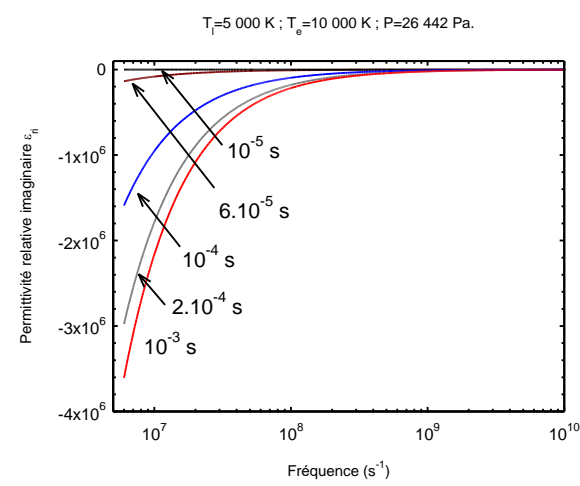

Figure 4. Évolution des permittivités relatives, partie réelle à gauche et partie imaginaire à droite, en fonction de la pression et de l'état d'équilibre du plasma : a) Pression atmosphérique à l'équilibre thermique ; b) Pression atmosphérique hors de l'équilibre thermique; c) Pression à $10 \mathrm{~km}$ d'altitude à l'équilibre thermique ; d) Pression à $10 \mathrm{~km}$ d'altitude hors de l'équilibre thermique. 
Les sections efficaces de collision entre les électrons et les particules chargées dépendent des concentrations en particules chargées au travers de la longueur de Debye [21]. La longueur de Debye diminue lorsque la température augmente. L'écrantage autour des particules chargées devient alors plus efficace. Ainsi, les sections efficaces diminuent lorsque les concentrations en particules chargées augmentent. Lorsque le déséquilibre thermique est pris en compte, nous pouvons décrire plusieurs phases successives pour le calcul des fréquences de collision. Dans un premier temps ( $<410^{-5} \mathrm{~s}$ pour $0 \mathrm{~km}$ d'altitude et $<10^{-4} \mathrm{~s}$ pour $10 \mathrm{~km}$ d'altitude), les collisions les plus contributives sont les collisions entre les électrons et les particules neutres, puis lorsque les particules chargées sont en nombre suffisant les collisions entre les électrons et les particules chargées. Ainsi, dans le cas d'un plasma déséquilibré thermiquement, on retrouve au niveau de la valeur absolue des permittivités relatives une augmentation de la permittivité puis une diminution pour atteindre un niveau d'équilibre. Dans le cas des plasmas équilibrés thermiquement et pour la température de $5000 \mathrm{~K}$ considérée, les concentrations électroniques sont trop faibles pour que la contribution des collisions entre les électrons et les particules chargées soit détectable.

\section{Réalisations numériques : cas tests}

\subsection{Propriétés diélectriques du plasma}

Afin de mettre en exergue l'influence des propriétés diélectriques du plasma et de l'onde incidente, au travers de sa fréquence d'excitation, nous représentons la valeur absolue de la permittivité réelle et imaginaire exprimée en décibel (voir les relations (14) et (15) dans la suite) en fonction du temps et en fonction de la fréquence de l'onde incidente comprise entre $10 \mathrm{MHz}$ et $10 \mathrm{GHz}$ sur les cartographies des Figures 5 à 8 .

Compte tenu des échelles de valeurs observées (voir la Figure 4) concernant les parties réelle et imaginaire du module de la permittivité diélectrique relative, les Figures 5 et 7 (pour la partie réelle) et les Figures 6 et 8 (pour la partie imaginaire) sont obtenues à partir des relations (14) et (15) :

et

$$
|\operatorname{Re}(e p s)|_{d B}=20 \cdot \log _{10}\left(\left|\operatorname{Re}\left(\varepsilon_{r}\right)\right|\right) \quad,
$$

$$
|\operatorname{Im}(e p s)|_{d B}=20 \cdot \log _{10}\left(\left|\operatorname{Im}\left(\varepsilon_{r}\right)\right|\right) \quad,
$$

où $\varepsilon_{r}$ est le permittivité diélectrique relative complexe calculée précédemment, les termes $\operatorname{Re}($.) et $\operatorname{Im}($.) sont associés aux parties réelles et imaginaire d'un nombre complexe. Les formules précédentes sont exprimées en décibels (dB).

La Figure 5 donne l'évolution de la partie réelle de la permittivité diélectrique, exprimée en $\mathrm{dB}$, du plasma en fonction du temps et de la fréquence d'excitation de l'onde considérée. À l'altitude $0 \mathrm{~km}$ (Figure 5 en haut), on constate que l'évolution est forte entre les instants $t_{0}=10 \mu \mathrm{s}$ et $t_{1}=1 \mathrm{~ms}$ où la partie réelle $|\operatorname{Re}(\mathrm{eps})|_{d B}$ de la permittivité exprimée en décibel passe de $0 \mathrm{~dB}$ à $20 \mathrm{~dB}$. Puis à partir d'une milliseconde, les valeurs convergent vers des valeurs stables (> $30 \mathrm{~dB}$ ) pour la gamme de fréquence prise en compte. En observant la cartographie à l'altitude de $10 \mathrm{~km}$ d'altitude (Figure 5 en bas), on observe que pour les instants autour de $0,1 \mathrm{~ms}$ on note une diminution de la permittivité exprimée en décibel qui est plus marquée pour les fréquences d'excitation proches de $2 \mathrm{GHz}$. Puis, en fonction du temps, les valeurs augmentent et convergent vers des valeurs stables $(>20 \mathrm{~dB}$ ). Ainsi, le comportement de la partie réelle de la permittivité diélectrique est fortement impacté par l'altitude à laquelle le plasma est produit. 

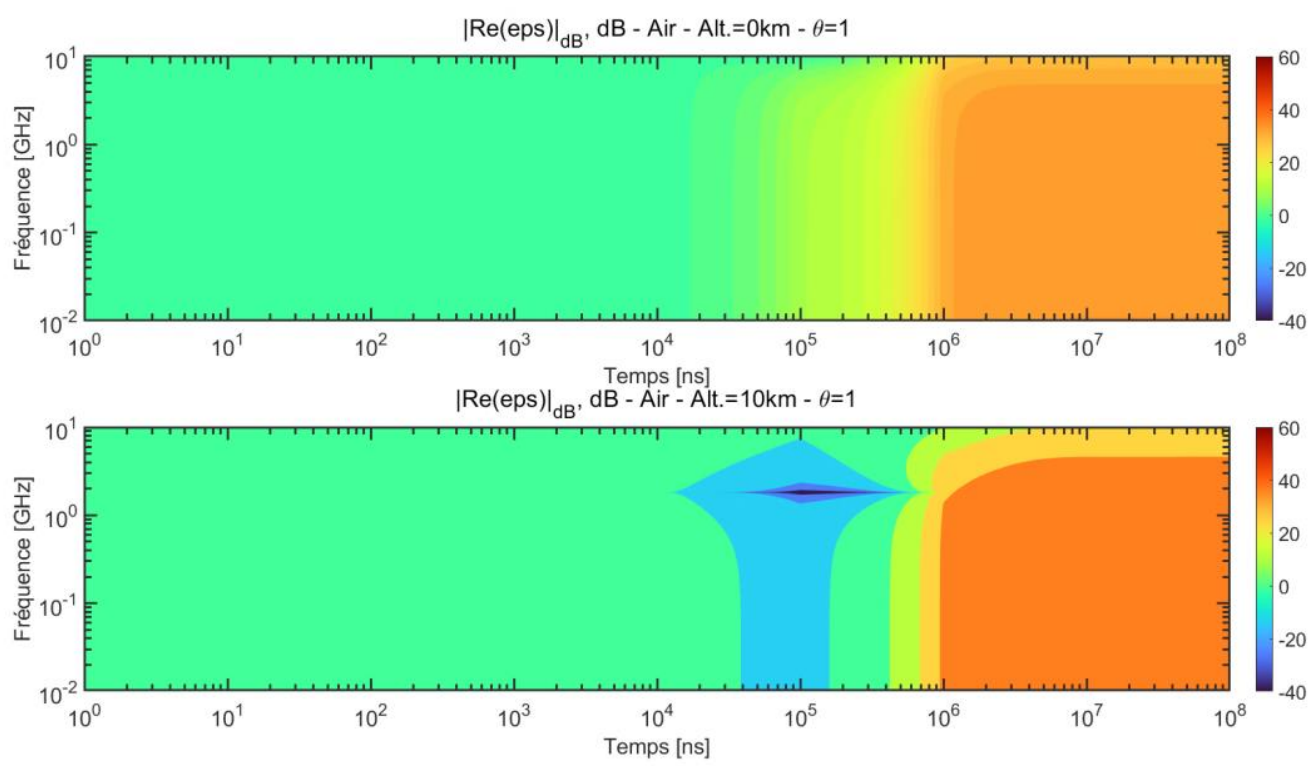

Figure 5. Évolution de la composante réelle, de la permittivité diélectrique du plasma à l'altitude $0 \mathrm{~km}$ (en haut) et à $10 \mathrm{~km}$ (en bas) à l'équilibre thermique $\left(T_{e}=T_{l}\right)$; pour une gamme de fréquences de l'onde incidente allant de $10 \mathrm{MHz}$ à $10 \mathrm{GHz}$ et pour un temps de simulation allant de $1 \mathrm{~ns}$ à $100 \mathrm{~ms}$.
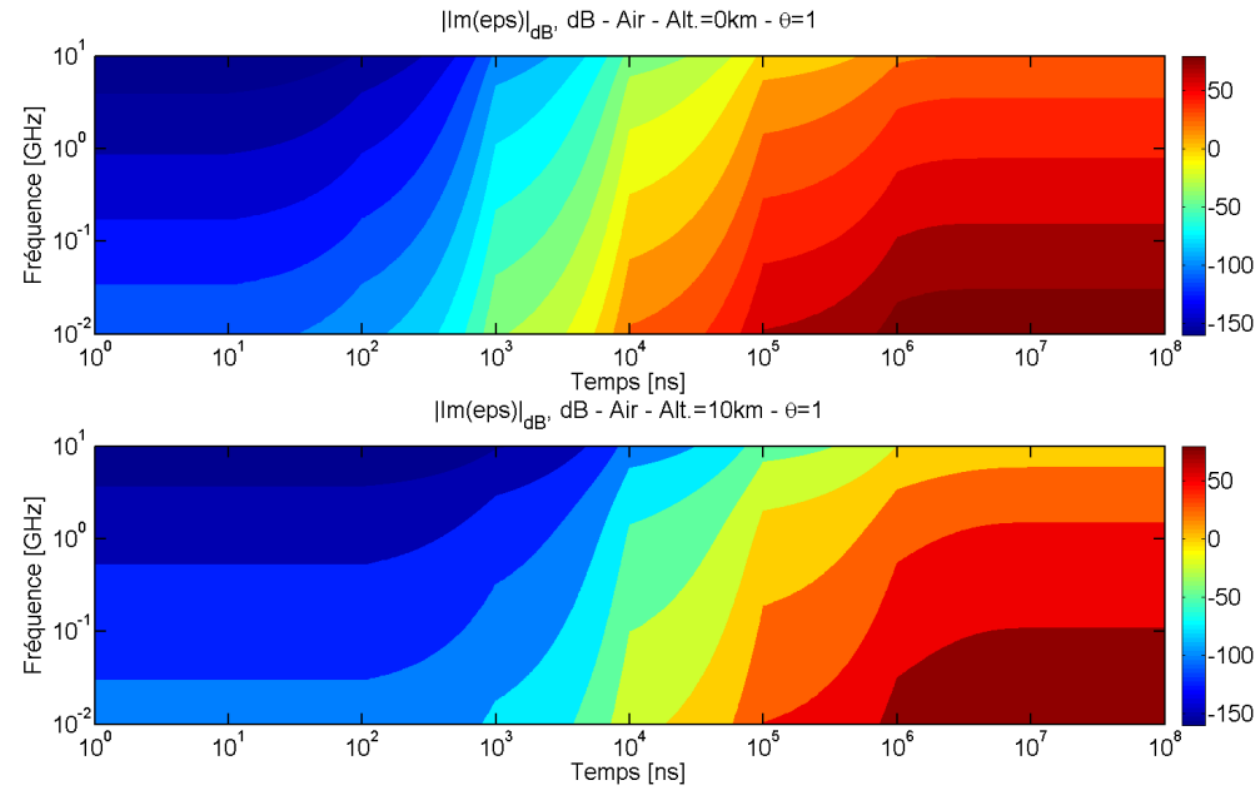

Figure 6. Évolution de la composante imaginaire de la permittivité diélectrique du plasma à l'altitude $0 \mathrm{~km}$ (en haut) et à $10 \mathrm{~km}$ (en bas) à l'équilibre thermique $\left(T_{e}=T_{l}\right)$; pour une gamme de fréquences de l'onde incidente allant de $10 \mathrm{MHz}$ à $10 \mathrm{GHz}$ et pour un temps de simulation allant de $1 \mathrm{~ns}$ à $100 \mathrm{~ms}$. 
La Figure 6 donne l'évolution de la partie imaginaire de la permittivité diélectrique du plasma en fonction du temps et de la fréquence d'excitation de l'onde considérée, ceci à l'équilibre thermique et pour les deux altitudes considérées. Pour les deux altitudes, on constate une forte évolution entre les instants $t_{0}=50 \mathrm{~ns}$ et $t_{1}=5 \mathrm{~ms}$ où la partie imaginaire $|\operatorname{Im}(\mathrm{eps})|_{d B}$ de la permittivité exprimée en décibel passe de $-150 \mathrm{~dB}$ à $60 \mathrm{~dB}$. On constate ainsi que l'évolution temporelle de la partie imaginaire est importante avec une variation de l'ordre de $210 \mathrm{~dB}$. Puis, en fonction du temps les valeurs augmentent et convergent vers des valeurs stables à partir de $5 \mathrm{~ms}$. Cependant, on notera une dynamique très importante sur toute la gamme de fréquence d'excitation considérée de l'onde incidente. Par exemple à 10 ns ou à $0,1 \mathrm{~s}$, on obtient aux alentours de $100 \mathrm{~dB}$ de dynamique. De plus, on peut noter que le comportement de la partie imaginaire de la permittivité diélectrique est largement moins impacté par l'altitude à laquelle le plasma est produit comparativement (Figure 6 en haut et en bas) au cas de la partie réelle précédemment évoqué (Figure 5).
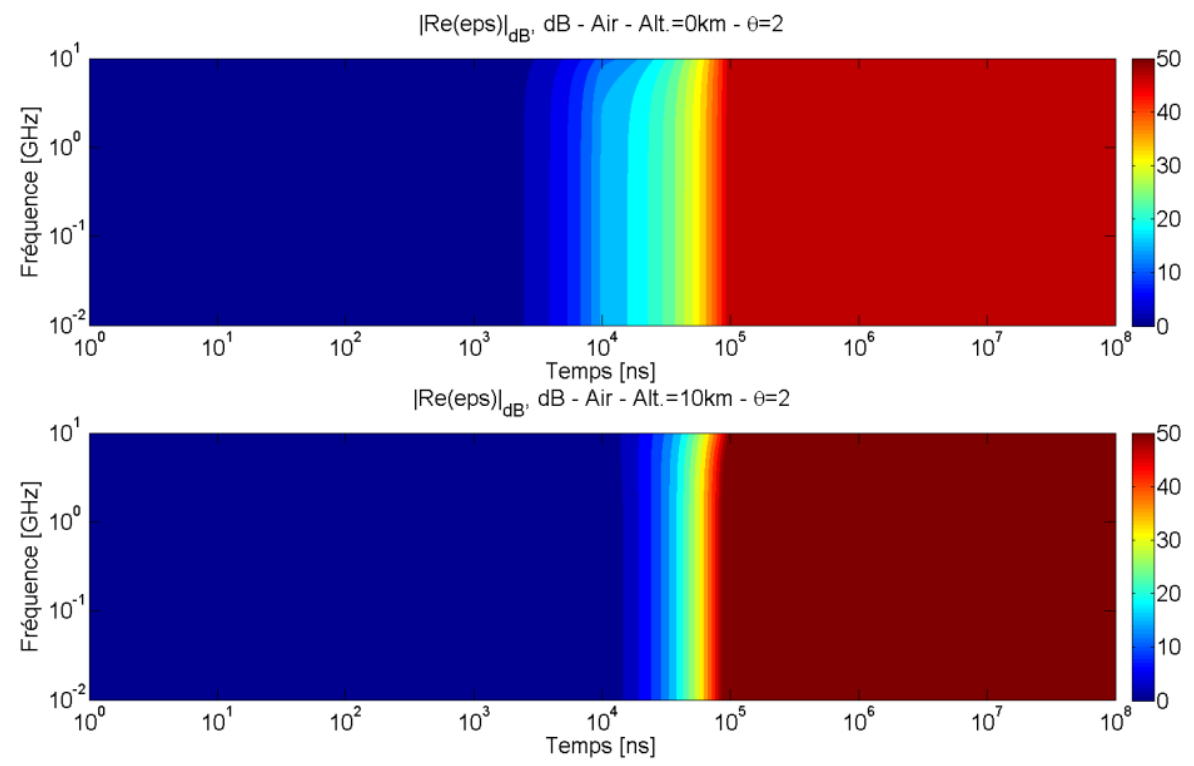

Figure 7. Évolution de la composante réelle de la permittivité diélectrique du plasma à l'altitude $0 \mathrm{~km}$ (en haut) et à $10 \mathrm{~km}$ (en bas) au déséquilibre thermique $(\theta=2)$; pour une gamme de fréquences allant de $10 \mathrm{MHz}$ à $10 \mathrm{GHz}$ et un temps de simulation allant de $1 \mathrm{~ns}$ à $100 \mathrm{~ms}$.

Les Figures 7 et 8 donnent les évolutions de la partie réelle et imaginaire de la permittivité diélectrique du plasma en fonction du temps et de la fréquence d'excitation de l'onde considérée, le plasma étant considéré hors de l'équilibre thermique et pour les deux altitudes considérées. L'évolution de la composante réelle de la permittivité diélectrique détaillée dans la Figure 7 montre un comportement similaire en fonction du temps et sur la gamme de fréquence considérée entre le cas de l'altitude de $0 \mathrm{~km}$ (en haut) et celui de $10 \mathrm{~km}$ (en bas). On constate un gradient très important pour les instants compris entre $1 \mathrm{~ms}$ et $100 \mathrm{~ms}$. Ce gradient est de l'ordre de $50 \mathrm{~dB}$ qui est nettement supérieur au gradient obtenu dans le cas du plasma à l'équilibre thermique (Figure 5). 
Comparativement au cas de l'équilibre thermique (Figure 6), la Figure 8 montre l'évolution de la partie imaginaire de la permittivité diélectrique du plasma hors de l'équilibre thermique. On note une dynamique plus importante passant de $-150 \mathrm{~dB}$ à $100 \mathrm{~dB}$ dans la gamme de fréquence considérée. L'évolution de la partie imaginaire étant plus importante entre 10 ns et $0.1 \mathrm{~ms}$ à l'altitude de $0 \mathrm{~km}$ et entre $100 \mathrm{~ns}$ et $1 \mathrm{~ms}$ à $10 \mathrm{~km}$. Les valeurs de la partie imaginaire de la permittivité diélectrique se stabilisant pour les temps supérieurs.
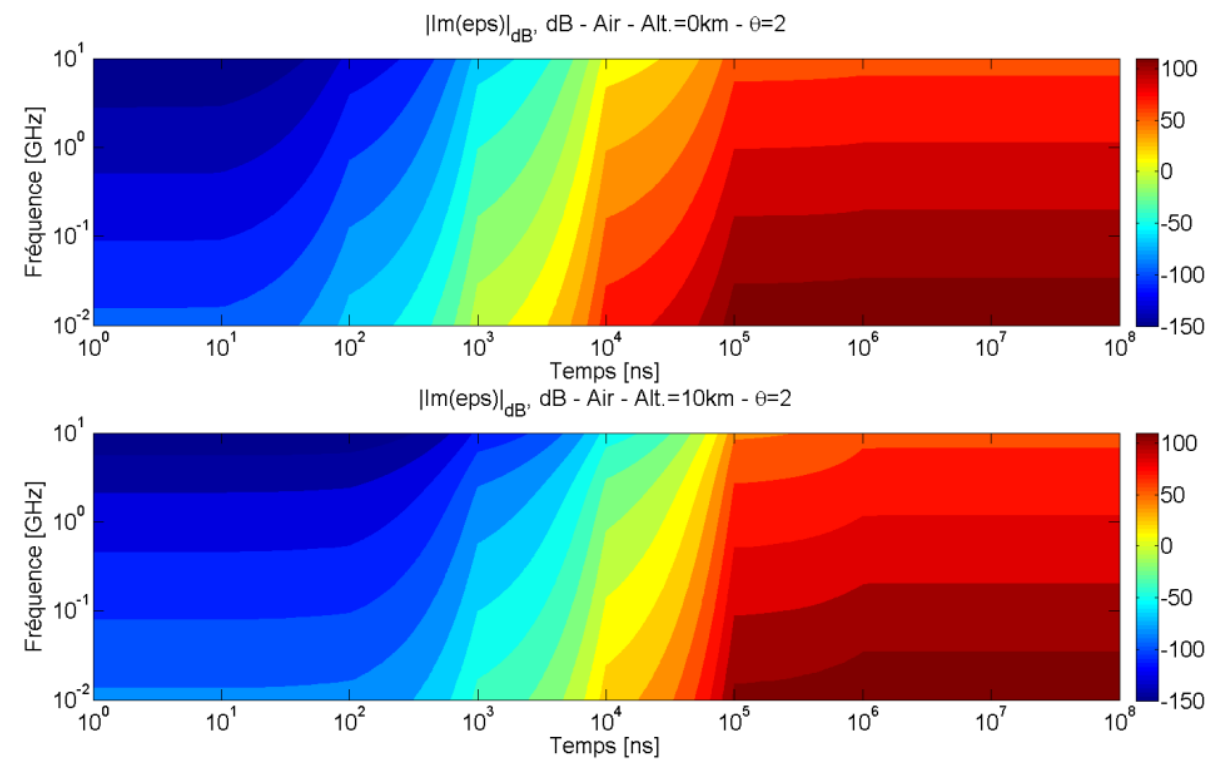

Figure 8. Évolution de la composante imaginaire de la permittivité diélectrique du plasma à

l'altitude $0 \mathrm{~km}$ (en haut) et à $10 \mathrm{~km}$ (en bas) au déséquilibre thermique $(\theta=2)$; pour une gamme de fréquences allant de $10 \mathrm{MHz}$ à $10 \mathrm{GHz}$ et un temps de simulation allant de $1 \mathrm{~ns}$ à $100 \mathrm{~ms}$.

Ainsi, les Figures 5 à 8 montrent une grande variabilité des permittivités réelles et imaginaires en fonction de l'altitude, du temps et de la fréquence de l'onde incidente. Afin de mettre en évidence l'influence la permittivité sur la transmission et la réflexion d'une onde incidente, nous considérons deux cas tests numériques. Dans le premier cas, nous prenons en compte une couche de plasma insérée entre deux espaces libres et dans le second cas étudié nous considérons une couche de plasma accolée à une plaque de métal parfait. 


\subsection{Description du cas test \#1}

Dans le premier cas test étudié, nous considérons une couche de plasma insérée entre deux espaces libres. La Figure 9 présente le cas test\#1 concernant la transmission et la réflexion de l'onde électromagnétique dans l'espace libre. La couche de plasma considérée dans la suite correspond à une épaisseur de $2 \mathrm{~mm}$.

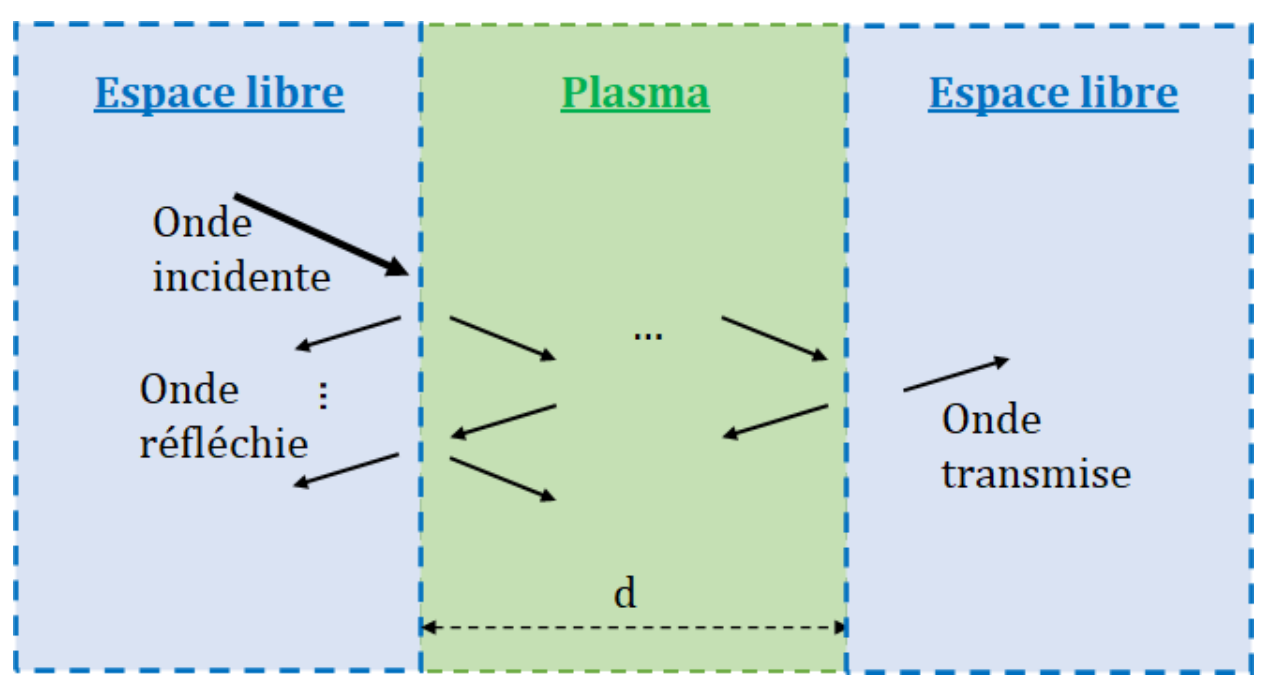

Figure 9. Descriptif du cas test \#1 proposé : le niveau de l'onde transmise est caractérisé par le coefficient $T$; le niveau de l'onde réfléchie est caractérisé par le coefficient $R, d=2 \mathrm{~mm}$.

Sur la base du schéma générique présenté dans la Figure 9, on considérera à nouveau dans la suite le cas d'une onde plane incidente, normale à la couche plasma (considérée comme semiinfinie).

S'appuyant sur le cas test décrit dans la Figure 9, les niveaux des champs électromagnétiques transmis et réfléchi sont donnés à partir des développements analytiques donnés décrits dans [1] :

$$
\begin{aligned}
& t=2 \sqrt{\varepsilon_{r}} e^{i k_{0} d} / 2 \sqrt{\varepsilon_{r}} \cosh \left(i k_{p} d\right)+\left(\varepsilon_{r}+1\right) \sinh \left(i k_{p} d\right) \\
& r=1-\varepsilon_{r} / 2 \sqrt{\varepsilon_{r}} \operatorname{coth}\left(i k_{p} d\right)+\varepsilon_{r}+1
\end{aligned}
$$

où $\varepsilon_{r}$ est la permittivité diélectrique relative complexe (11) du milieu plasma, $d$ est l'épaisseur du plasma, $k_{0}$ et $k_{p}$ sont les nombres d'onde respectivement dans l'air et le plasma, les coefficients $r$ et $t$ sont les coefficients de transmission et de réflexion.

Dans la suite, on s'intéressera aux grandeurs $T$ et $R$, sur la base des équations (16) et (17), telles que $: T=20 \log _{10}|t|$ et $R=20 \log _{10}|r|($ en $\mathrm{dB})$. 


\subsection{Résultats numériques cas test \#1}
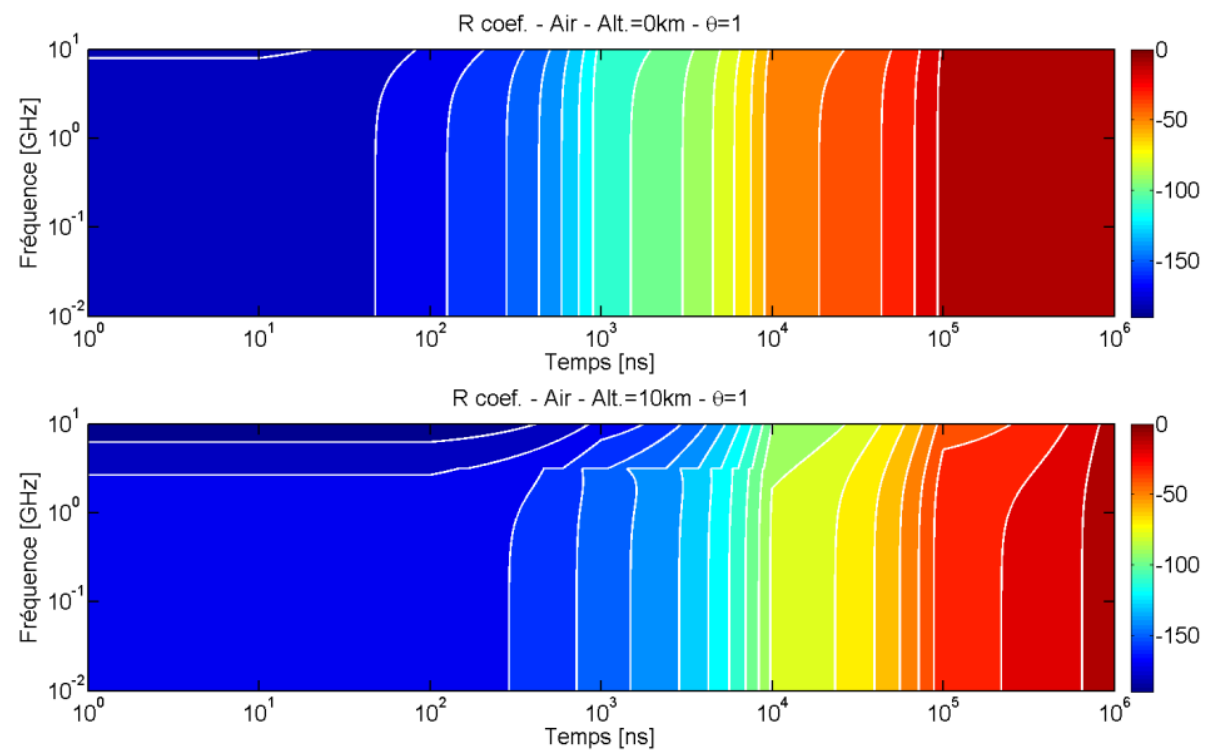

Figure 10. Coefficient de réflexion $R$ pour un plasma d'air à l'altitude de $0 \mathrm{~km}$ et à l'équilibre thermique $(\theta=1)$; pour une gamme de fréquences allant de $10 \mathrm{MHz}$ à $10 \mathrm{GHz}$ et un temps de simulation allant de $1 \mathrm{~ns}$ à $1 \mathrm{~ms}$.

La Figure 10 montre l'évolution du coefficient de réflexion $R$ observé sur une couche de plasma à l'équilibre thermique $(\theta=1)$ d'épaisseur $d$ de $2 \mathrm{~mm}$ pour les deux altitudes considérées. La représentation se limite aux temps inférieurs au temps où les valeurs de permittivité diélectrique atteignent leur stabilité soit $t_{0}=1 \mathrm{~ms}$ (cf $\S 2.1$ ). On constate que le coefficient de réflexion reste très faible (i.e. inférieur à $-10 \mathrm{~dB}$ ) jusqu'à ce que les permittivités diélectriques atteignent leur valeur de stabilité $(\$ 2.1)$ correspondant au moment où le plasma atteint l'équilibre chimique obtenu vers $t_{0}=1 \mathrm{~ms}$ (§1.3.).

La Figure 11 représente le coefficient de réflexion $R$ lorsque le plasma est hors de l'équilibre thermique $(\theta=2)$. À la différence de l'équilibre thermique, le coefficient de réflexion atteint une valeur stable plus rapidement c'est-à-dire vers l'instant $t_{0}=10 \mu \mathrm{s}$ et $t_{0}=100 \mu \mathrm{s}$ respectivement pour une altitude respectivement de $0 \mathrm{~km}$ (Figure 11 en haut) et $10 \mathrm{~km}$ (Figure 11 en bas). Pour les temps inférieurs à ces moments-là, le coefficient de réflexion $R$ est inférieur à $-10 \mathrm{~dB}$ puis devient supérieur lorsque le plasma atteint l'équilibre chimique (\$1.3.). Comparativement au cas de l'équilibre thermique $(\theta=1)$ de la Figure 10, la dynamique reste similaire pour le cas en déséquilibre thermique (environ de $-190 \mathrm{~dB}$ à $0 \mathrm{~dB}$ ). Cependant, on peut noter, qu'entre ces deux états d'équilibre thermique, le comportement du coefficient de réflexion $R$ est différent avant l'établissement d'une valeur de coefficient de réflexion stable $(R=0 d B)$. 

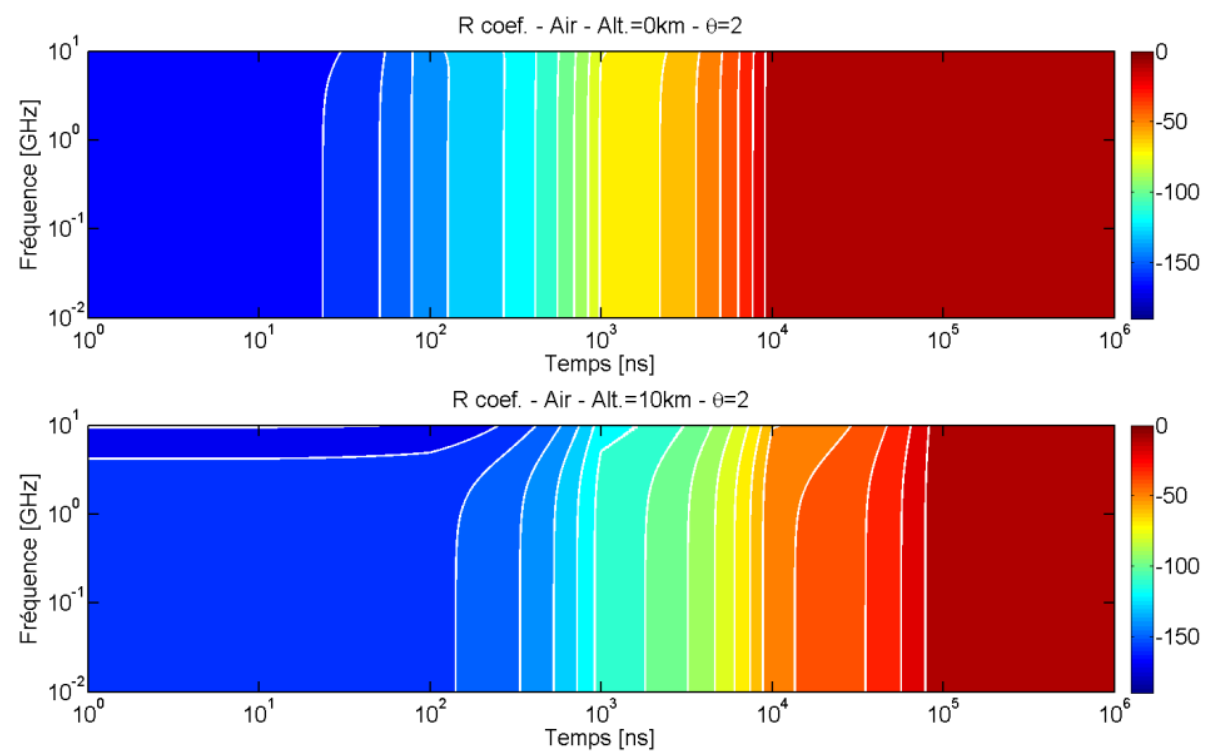

Figure 11. Coefficient de réflexion $R$ pour un plasma d'air à l'altitude de $0 \mathrm{~km}$ et au déséquilibre thermique $(\theta=2)$; pour une gamme de fréquences allant de $10 \mathrm{MHz}$ à $10 \mathrm{GHz}$ et un temps de simulation allant de $1 \mathrm{~ns}$ à $1 \mathrm{~ms}$.
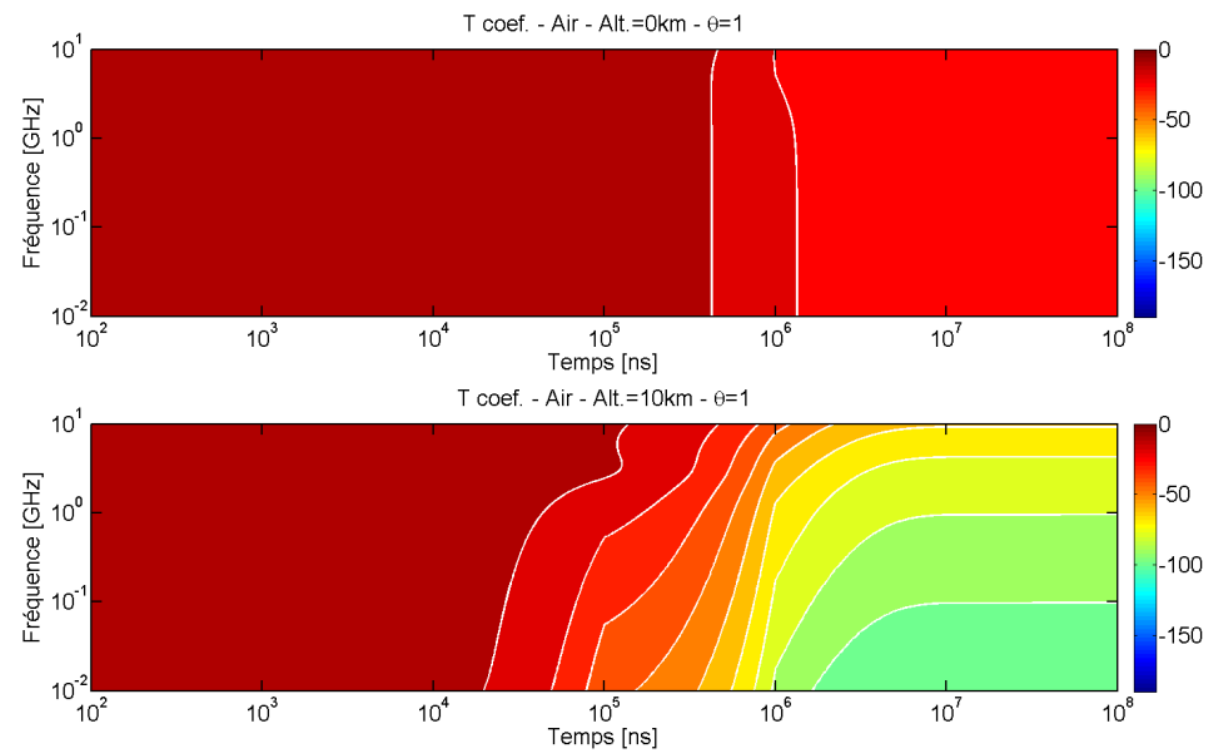

Figure 12. Coefficient de transmission $T$ pour un plasma d'air à l'altitude de $0 \mathrm{~km}$ et à l'équilibre thermique $(\theta=1)$; pour une gamme de fréquences allant de $10 \mathrm{MHz}$ à $10 \mathrm{GHz}$ et un temps de simulation allant de $100 \mathrm{~ns}$ à $100 \mathrm{~ms}$.

La Figure 12 montre l'évolution du coefficient de transmission à travers la couche plasma à l'équilibre thermique et d'épaisseur $d$ de $2 \mathrm{~mm}$. Les valeurs du coefficient réflexion deviennent stables pour des temps de l'ordre de $t_{0}=1 \mathrm{~ms}$ pour une altitude de $0 \mathrm{~km}$ et de l'ordre de $t_{0}=$ $5 \mathrm{~ms}$ pour une altitude de $10 \mathrm{~km}$. Ces dernières valeurs temporelles sont en accord avec les résultats qui concernent les coefficients de réflexion obtenus dans la Figure 10. Cependant, on notera un comportement différent pour le plasma formé à l'altitude de $0 \mathrm{~km}$ (Figure 12 en haut) et celui formé à $10 \mathrm{~km}$ (Figure 12 en bas). Ceci peut s'expliquer par la différence de 
comportement entre les permittivités calculées pour ces deux altitudes (Figures 5 et 6 ). Ainsi, au-delà de $t_{0}=1 \mathrm{~ms}$, la part de l'onde transmise est extrêmement faible. Le modèle proposé permet de suivre l'évolution temporelle de ce coefficient en fonction du temps et de la fréquence de l'onde transmise.
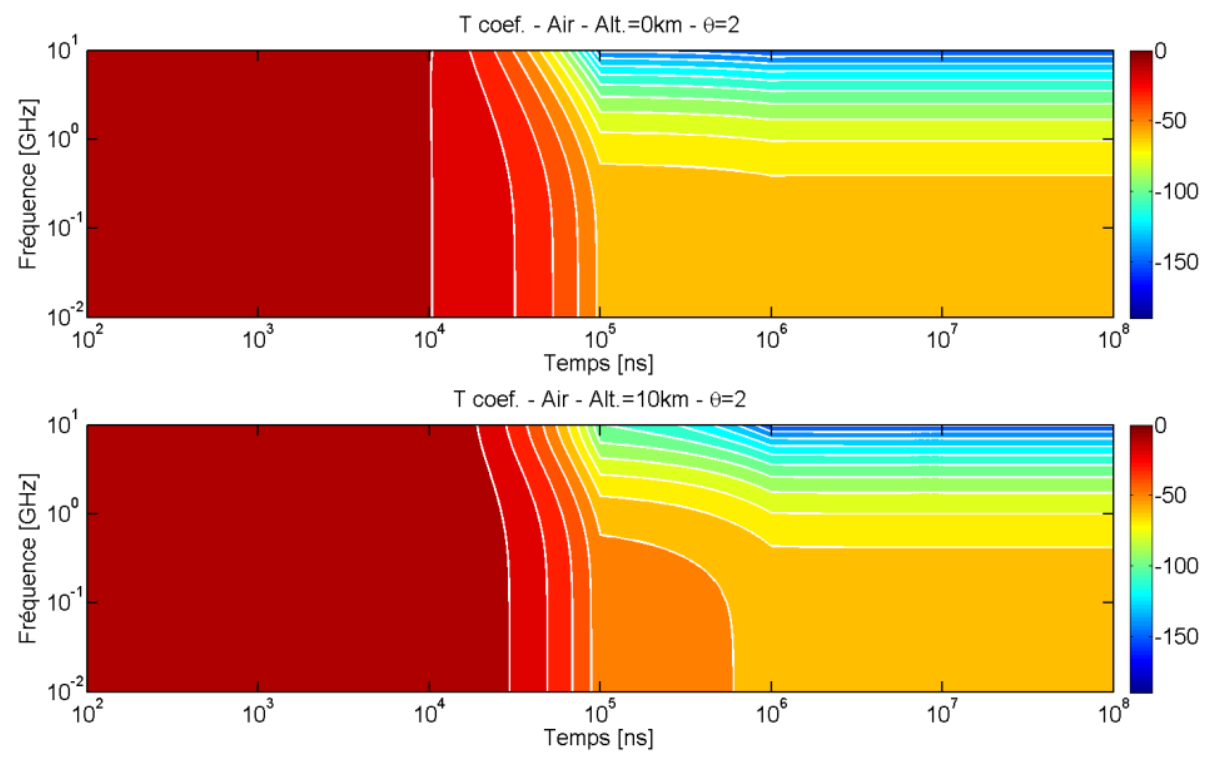

Figure 13. Coefficient de transmission $T$ pour un plasma d'air à l'altitude de $0 \mathrm{~km}$ et au déséquilibre thermique $(\theta=2)$; pour une gamme de fréquences de l'onde incidente allant de $10 \mathrm{MHz}$ à $10 \mathrm{GHz}$ et un temps de simulation allant de $100 \mathrm{~ns}$ à $100 \mathrm{~ms}$.

La Figure 13 traduit l'évolution du coefficient de transmission à travers une couche de plasma hors de l'équilibre thermique et pour deux altitudes. Comme dans le cas précèdent au-delà des temps de $t_{0}=10 \mu \mathrm{s}$ pour l'altitude de $0 \mathrm{~km}$ et pour $t_{0}=100 \mu \mathrm{s}$ pour l'altitude de $10 \mathrm{~km}$, le coefficient de transmission devient faible $(<-75 \mathrm{~dB})$. Nous avons alors une forte atténuation du signal transmis et ce plus particulière pour les fréquences de l'onde incidente supérieure à $1 \mathrm{GHz}$.

\subsection{Description du cas test \#2}

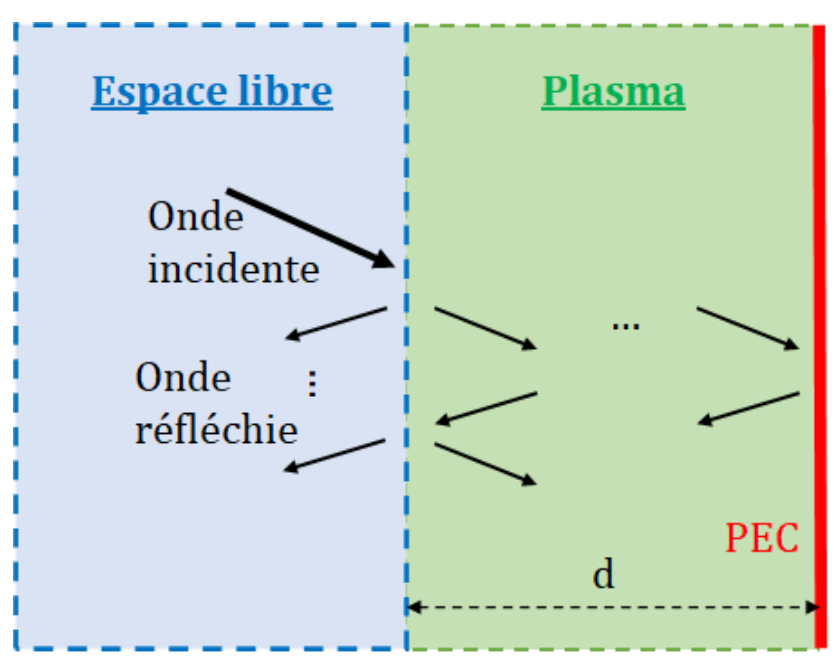

Figure 14. Configuration sous test pour le cas test \#2. 
Dans le second cas de test étudié, nous considérons une couche de plasma de $200 \mathrm{~mm}$ d'épaisseur accolée à une plaque de métal parfait (PEC). Ce cas test\#2 est présenté sur la Figure 14. Contrairement au cas test \#1, nous nous intéresserons uniquement au coefficient de réflexion. En effet, aucune transmission n'étant autorisée au-delà de la couche de métal parfait, (PEC). On considérera à nouveau dans la suite le cas d'une onde plane incidente, normale à la couche plasma (considérée comme semi-infinie).

Le coefficient de réflexion $r_{p e c}$ est donné par la relation :

$$
r_{p e c}=\left|Z_{\text {in }}-1\right| /\left|Z_{\text {in }}+1\right|
$$

avec :

$$
Z_{\text {in }}=\sqrt{\frac{\mu_{r}}{\varepsilon_{r}}} \tanh \left(i k_{p} d \sqrt{\mu_{r}}\right)
$$

Cette relation est obtenue à partir des travaux de Shen et al issus de [35]. Elle tient compte de la désadaptation entre le milieu de propagation (espace libre $Z_{0}$ ) et la couche de plasma considérée d'épaisseur $d$ dont l'impédance de milieu $Z_{\text {in }}$ est donnée par la relation (19) avec $\mu_{r}$ la perméabilité magnétique relative prise égale à 1 car nous avons supposé le plasma non magnétique.

\subsection{Résultats numériques cas test \#2}
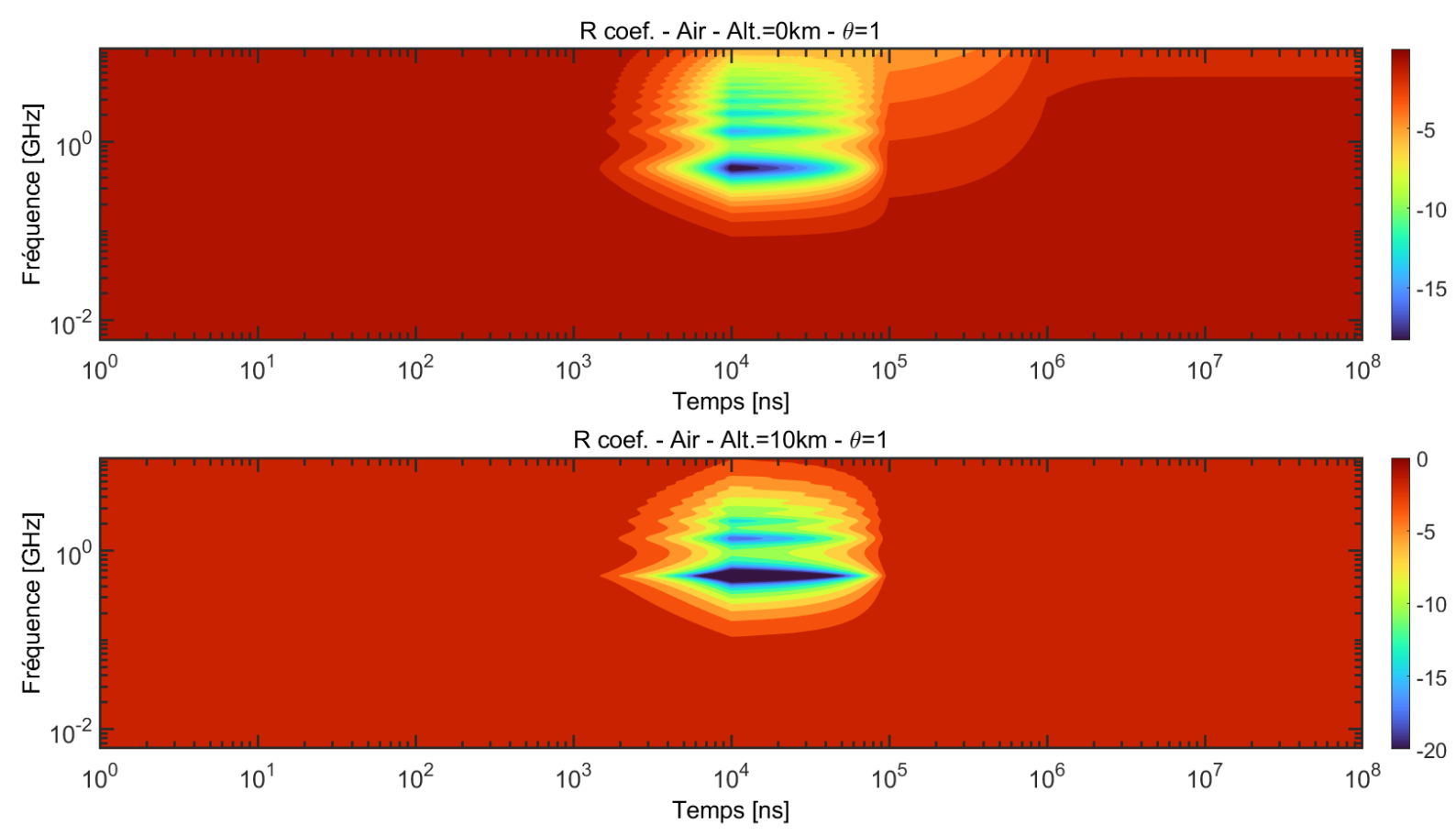

Figure 15. Coefficient de réflexion $\mathrm{R}$ (cas test \#2) pour un plasma d'air à l'altitude $0 \mathrm{~km}$ (en haut) et $10 \mathrm{~km}$ (en bas), à l'équilibre thermique $(\theta=1)$; pour une gamme de fréquences allant de $10 \mathrm{MHz}$ à $10 \mathrm{GHz}$ et un temps de simulation allant de $1 \mathrm{~ns}$ à $100 \mathrm{~ms}(\mathrm{~d}=200 \mathrm{~mm})$. 
La Figure 15 illustre l'évolution du coefficient de réflexion obtenu dans le cas de la configuration de test \#2. Cette réflexion est essentiellement due à la présence de la limite parfaitement conductrice. Les résultats obtenus sont en accord avec les attentes du dispositif numérique proposé : forte réflexion sur une majorité des points définis dans le plan d'expérience. Ainsi, on note un coefficient de réflexion proche de $0 \mathrm{~dB}$ sur l'ensemble de la gamme temporelle et pour les fréquences d'excitation de l'onde incidente prises en compte. Cependant, on constate un comportement particulier aux alentours de l'instant $t_{0}=10 \mu \mathrm{s}$ dans le cas du plasma à l'équilibre thermique (Figure 15), le coefficient de réflexion devient extrêmement faible (i.e. inférieur à $-10 \mathrm{~dB}$ ), ceci pour une gamme de fréquence allant de 300 $\mathrm{MHz}$ à $6 \mathrm{GHz}$.

La Figure 16 montre l'évolution du coefficient de réflexion obtenu dans le cas test \#2 pour le plasma hors de l'équilibre thermique $\theta=2$. On constate des valeurs du coefficient de réflexion qui restent notables, comprises entre $-3 \mathrm{~dB}$ et $0 \mathrm{~dB}$, sur toute la gamme de fréquence et pour toute la gamme temporelle considérée pour l'altitude de $0 \mathrm{~km}$ (Figure 16 en haut). Nous n'avions pas noté ce comportement dans le cas du plasma à l'équilibre thermique (Figure 15 en haut). Dans le cas du plasma hors de l'équilibre pour l'altitude $10 \mathrm{~km}$ (Figure 16 en bas), on note une zone marquante avec un coefficient de réflexion faible (compris entre $-20 \mathrm{~dB}$ et $-10 \mathrm{~dB}$ ), ceci pour $t_{0}=10 \mu \mathrm{s}$ et une gamme de fréquences allant de $300 \mathrm{MHz}$ à $4 \mathrm{GHz}$.
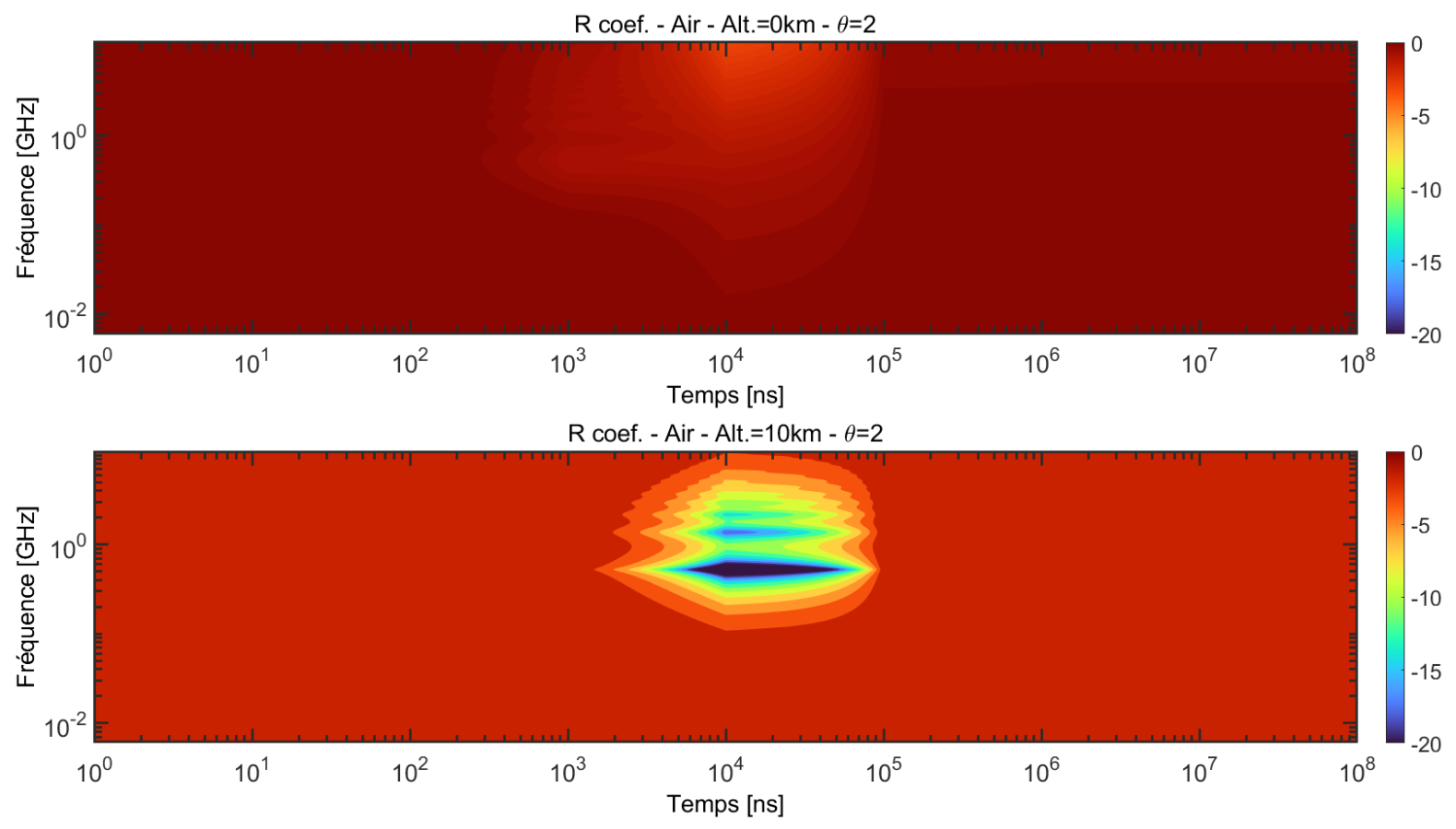

Figure 16. Coefficient de réflexion $R$ (cas test \#2) pour un plasma d'air à l'altitude $0 \mathrm{~km}$ (en haut) et $10 \mathrm{~km}$ (en bas), au déséquilibre thermique $(\theta=2)$; pour une gamme de fréquences allant de $10 \mathrm{MHz}$ à $10 \mathrm{GHz}$ et un temps de simulation allant de $1 \mathrm{~ns}$ à $100 \mathrm{~ms}$ (d=200mm). 


\section{Conclusion}

Dans cet article, nous avons tout d'abord rappelé l'ensemble des formulations nécessaire aux calculs des concentrations des espèces chimiques dans un plasma à l'équilibre et hors de l'équilibre thermique en fonction du temps. Puis, nous avons rappelé les formulations nécessaires aux calculs de la permittivité et discuté du choix des fréquences individuelles de collision entre les électrons et les espèces chimiques électriquement neutres nécessaires aux calculs. Nous avons ensuite étudié l'évolution des concentrations chimiques pour un plasma d'air à l'équilibre thermique et hors de l'équilibre thermique. Ainsi, nous avons pu établir deux temps d'équilibre chimique, l'un plus rapide pour les espèces majoritaires et l'autre plus lent pour les espèces chimiques minoritaires.

Bien que nous ayons émis des hypothèses fortes afin de réaliser les calculs, pas de diffusion de particules, pas de convection, aucuns dipôles créés par l'onde électromagnétique incidente et un plasma non-magnétique. Ces hypothèses semblent valides lors de la création d'un plasma transitoire par exemple lors de la décharge de foudre. L'un des points les plus marquant est que les résultats montrent que le plasma passe de la permittivité du gaz à celle d'un métamatériau. Ce point a déjà été montré par Fantini et al. [10] en rapprochant le comportement d'un plasma placé dans un résonateur à celui d'un métamatériau de type « main gauche ». Ainsi, des métamatériaux seraient susceptibles d'exister dans la nature.

On constate également que la confrontation du modèle de permittivité diélectrique considéré dans ces travaux a permis de mettre en œuvre l'effet de la stimulation en incidence normale de couches d'épaisseurs variées concernant deux cas tests distincts reprenant deux cas génériques : traversée d'une paroi plasma entre deux milieux d'espace libre (cas test\#1), et réflexion sur une plaque parfaitement conductrice recouverte par une couche plasma (cas test \#2). L'analyse de l'évolution de la cinétique chimique a permis d'extraire des informations pertinentes à la fois pour les cas tests \#1 et \#2 sur le coefficient de réflexion de la couche plasma en fonction du temps et de la fréquence de stimulation. L'étude du cas test \#1 a également permis de définir les niveaux de champs électromagnétiques transmis à travers la couche plasma.

Le modèle proposé autorise des travaux futurs concernant les effets de la propagation d'ondes électromagnétiques dans le cadre de la formation d'un milieu plasma pour des configurations réalistes. Ces travaux montrent qu'à partir de la mesure des coefficients de réflexion d'ondes électromagnétiques nous pourrions estimer les dynamiques d'équilibre chimique de matériaux variés comme les plasmas. Des travaux sont actuellement en cours pour qualifier les niveaux de réflexion et de transmission des champs électromagnétiques dans le cadre d'applications en signature électromagnétique et en compatibilité des systèmes électroniques. 


\section{Références}

[1] L. Zheng, Q. Zhao, and X. J. Xing, (2015), « Effect of Plasma on Electromagnetic Wave Propagation and THz », ACES JOURNAL, Vol. 30, No. 11, p. 1241-1245.

https://aces-

society.org/includes/downloadpaper.php?of=ACES_Journal_November_2015_Paper_15 $\underline{\text { \&nf }}=15-11-15$

[2] R. Wang, X. Wang, S. Cheng, Y. Meng, G. Zhang, Y. Zhou, (2018), « Plasma passive jamming for SAR based on the resonant absorption effect ${ }^{\circ} \gg$, IEEE Trans. On Plasma Science, Vol. 46, No 4, p. 928-933.

DOI: https://doi.org/10.1109/TPS.2018.2811496

[3] J. Xu, B. Bai, Y. Zhu, Y-Y. Dong G. Zhao, (2017), « A novel plasma jamming technology based on the resonance absorption effect ${ }^{\circ} »$, IEEE Ant. And Wireless Propag. Letters, volume 16, p. 1056-1059.

DOI: https://doi.org/10.1109/LAWP.2016.2619671

[4] A. Shoory, F. Rachidi, M. Rubinstein, R. Thottappillil, (2011), « On the measurement and calculation of horizontal electric fields from lightning », IEEE Trans. On Electromagnetic Compatibility, volume 53, numéro 3, p. 792-801.

DOI: $\underline{\text { https://doi.org/10.1109/TEMC.2010.2093602 }}$

[5] M. Bäckström, U. Jordan, D. Andersson, A. V. Kim, M. Lisak, O. Landen, (2011), « Can intentional electrical discharges be used for HPM protection? », IEEE International Symposium on Electromagnetic Compatibility, Long Beach, CA, USA, p. 752-757.

DOI: https://doi.org/10.1109/ISEMC.2011.6038409

[6] R. Brun, (2012) "High Temperature Phenomena in Shock Waves », Ouvrage collectif, Shock Wave Science and Technology Reference Library, Springer.

ISBN 978-3-642-25119-1

[7] V. Babrauskas, (2010), «Electric Arc Explosions », in Interflam 2010-Proc. 12th Intl. Conf., Interscience, p. 1283-1296, Communications Ltd, London

[8] E. L. Hill, J. D. Robb, (1968), « Pressure pulse from a lightning stroke », JGR, Vol 73, $\mathrm{N}^{\circ} 6$, p. $1883-1888$.

DOI: https://doi.org/10.1029/JB073i006p01883

[9] P. André, G Faure, A Mahfouf, S. Lalléchère, (2018), « Air plasma key parameters for electromagnetic wave propagation at and out of thermal equilibrium: applications to electromagnetic compatibility », ACES Journal, Vol 33, Nº 12, p. 1453-1460.

https://acessociety.org/includes/downloadpaper.php?of=ACES_Journal_December_2018_Paper_17 $\underline{\text { \&nf }=18-12-17}$

[10] L. Fantini, S. Dennison, H. Kim, M. Sarkarat, M. Lanagan, J. Hopwood, (2019), « Plasma reconfigurable metamaterial using $6.5 \mathrm{GHz}$ dielectric resonator array », Journal of Applied Physics, Vol 126, 203301, p. 1-12.

DOI: https://doi.org/10.1063/1.5121222

[11] C. Xu, N. Huret, M. Garnung and S. Celestin, « A New Detailed Plasma-Chemistry Model for the Potential Impact of Blue Jet Streamers on Atmospheric Chemistry », JGR Atmospheres, 125, e2019JD031789, p1-15.

DOI: https://doi.org/10.1029/2019JD031789 
[12]P. André, M.-A. Courty, A.K. Kagoné, Z. Koalaga, N. Kohio, F. Zougmoré, (2016), «Calcul de la composition chimique dans un plasma issu de mélanges de PTFE, d'air, de cuivre et de vapeur d'eau dans le cadre d'appareillages de coupure électrique à air ", JITIPEE, Vol 2, N¹, p. 3.1-3.18.

DOI: $\underline{\text { http://doi.org/10.18145/jitipee.v2i1.128.g70 }}$

[13] P. André, Y. Barinov, G. Faure, S. Shkol'nik, (2011), « Modelling radiation spectrum of a discharge with two liquid non-metallic (tap-water) electrodes in air at atmospheric pressure », Journal of Physics D: Applied Physics, vol. 44, n 37, p. 375203.

DOI: https://doi.org/10.1088/0022-3727/44/37/375203

[14] D. Staack, F. Bakhtier, A. Gutsol, A. Fridman, (2005), «Characterization of a dc atmospheric pressure normal glow discharge », Plasma Sources Science and Technology, vol. $14, \mathrm{n}^{\circ} 4$, p. $700-711$.

DOI: https://doi.org/10.1088/0963-0252/14/4/009

[15] T.-L. Zhao, Y. Xu, Y.H. Song, X.-S. Li, J.-L. Liu, J.-B. Liu et A.-M. Zhu, (2013), «Determination of vibrational and rotational temperatures in a gliding arc discharge by using overlapped molecular emission spectra », Journal of Physics D: Applied Physics, vol. $46, \mathrm{n}^{\circ} 34$, p. 345201.

DOI: https://doi.org/10.1088/0022-3727/46/34/345201

[16] P. André, (1995), «Partition Functions and Concentrations in Plasmas out of Thermal Equilibrium », IEEE transactions on Plasma Science, Vol 23, n 3 , p 453-458.

DOI: https://doi.org/10.1109/27.402339

[17] Y. Reuveni, Y. Yair, C. Price, G. Steinitz, (2017), « Ground level gamma-ray and electric field enhancements during disturbed weather: Combined signatures from convective clouds, lightning and rain », Atmospheric Research, Vol 196 p 142-150

DOI: https://doi.org/10.1016/j.atmosres.2017.06.012

[18] T. Marshall, M.P. McCarthy, W.D. Rust (1995), «Electric field magnitudes and lightning initiation in thunderstorms », Journal of geophysical research, vol 100, D4, p 7097-7103.

DOI: https://doi.org/10.1029/95JD00020

[19] P. André M. Abbaoui, (2017), « Déséquilibre thermique dans un plasma d'air ensemencé d'aluminium », JITIPEE, Vol. 3, n², p 3.1-3.10.

DOI: https://doi.org/10.18145/jitipee.v3i2.144

[20] J. Cen, P. Yuan, H. Qu, T. Zhang, (2011), « Analysis on the spectra and synchronous radiated electric field observation of cloud to-ground lightning discharge plasma », Physics of Plasmas, Vol. 18, p 113506.1-113506.6

DOI: https://doi.org/10.1063/1.3662106

[21]A.B. Murphy, (2000), «Transport coefficients of hydrogen and argon-hydrogen plasmas », PCPP., Vol. 20, p. 279-297.

DOI: https://doi.org/10.1023/a:1007099926249

[22] C. Park, J. T. Howe, R. L. Jaffe and G. V. Candler, (1994), « Review of chemical kinetic problems of future NASA missions. II - Mars entries », JTHT, vol. 8, n 1, p 9-23.

DOI: https://doi.org/10.2514/3.496

[23] J.P. Nougier, (2001), « Méthode de calcul numérique », Hermes Science Publications.

[24] A. Fridmann, L.A. Kennedy, (2004), « Plasma Physics and Engineering », Taylor et Francis, New York, London. 
[25] I.P. Shkarofsky, (1961), «Values of the transport coefficients in a plasma for any degree of ionization based on a Maxwellian distribution », Can J. Phys., p 1619-1703.

DOI: https://doi.org/10.1139/p61-192

[26] M. Capitelli, D. Bruno, A. Laricchiuta, (2013), «Fundamental Aspects of Plasma Chemical Physics - Kinetics », Vol 74, Springer Series on Atomic, Optical and Plasma Physics, Springer Edition.

ISBN 978-1-4419-8171-4

[27] P. André, L. Brunet, W. Bussière, J. Caillard, Lombard, J.P. Picard, (2004), « Transport Coefficients of Plasmas consisting of insulator vapours. Application to PE, POM, PMMA, PA66 and PC », Eur. Phys. J. Appl. Phys., volume 25, p. 169-183.

DOI: https://doi.org/10.1051/epjap:2004007

[28] Y. Itikawa, (1971), «Effective collision frequency of electrons in atmospheric gases », Planetary and Space Science, Vol., N 8, P. 993-1007.

DOI: $\underline{\text { https://doi.org/10.1016/0032-0633(71)90149-8 }}$

[29] Y. Itikawa, (1973), « Effective collision frequency of electrons in gases », The physics of fluids, Vol 16, $\mathrm{N}^{\circ} 6, \mathrm{p}$ 831-835.

DOI: $\underline{\text { https://doi.org/10.1063/1.1694435 }}$

[30] R.H. Neynaber, L.L. Marino, E.W. Rothe, W. Erhard, S.M. Trujillo, (1962), « Low-Energy Electron Scattering from Atomic Nitrogen », Phys. Rev. 129, pages 2069-2071.

DOI: https://doi.org/10.1103/PhysRev.129.2069

[31]B. Sourd, J. Aubreton, M-F. Elchinger, M. Labrot and U. Michon, (2006), « High temperature transport coefficients in e/C/H/N/O mixtures », J. Phys. D: Appl. Phys., Vol. 39, p. 1105-1119

DOI: https://doi.org/10.1088/0022-3727/39/6/016

[32] F.E. Spencer Jr, A.V. Phelps (1976), « Momentum Transfer cross-sections and conductivity integrals for gases of MHD interest », Inc. Proc. XVth Symp. Eng. Asp of MDH, Philadelphia PA, pages IX.9.1-IX.9.12.

[33] M.M. Fujimoto, M.T. Lee, (2000), «Elastic and absorption cross sections for electronnitric oxide collisions », J. Phys. B: At. Mol. Opt. Phys. Vol. 33, p 4759-4768

DOI: https://doi.org/10.1088/0953-4075/33/21/319

[34] M.J. Brunger, S.J. Buckman, (2002), « Electron-molecule scattering cross-sections. I. Experimental techniques and data for diatomic molecules », Physics Reports, Vol. 357, $\mathrm{N}^{\circ} 3-5, \mathrm{p} 215-458$.

DOI: https://doi.org/10.1016/S0370-1573(01)00032-1

[35] G. Shen, Z. Xu, Y. Li, (2006), « Absorbing properties and structural design of microwave absorbers based on W-type La-doped ferrite and carbon fiber composites », Journal of Magnetism and Magnetic Materials, Vol. 301, p 325-330.

DOI: https://doi.org/10.1016/j.jmmm.2005.07.007 